\title{
Comparison between Batwing versus Wise Pattern Mammoplasty for Upper Pole Breast Tumors
}

\author{
ASHRAF O.M. ALI, M.D.; EHAB H. ABD EL-WAHAB, M.D.; AHMED G. OTHMAN, M.D. and \\ ABD EL-RAHMAN M. ESSAM, M.Sc.
}

The Department of General Surgery, Faculty of Medicine, Ain Shams University

\begin{abstract}
Background: Since the recorded time, breast has been a symbol for motherhood, fiminity, and sexuality, it has been portrayed throughout history in works of art symbolizing to all those aspects of woman's life. So breasts in female life are described as "life giving and life destroying". Breast cancer existed in ancient times and reference to this disease can be found dating back as $3000 \mathrm{BC}$, in an Egyptian papyrus.

Aim of Study: The Aim of this study is to compare between batwing mammoplasty and inferiorly based wise pattern therapeutic mammoplasty in management of upper pole breast tumors regarding cosmetic results, oncological outcomes, rate of complications and degree of satisfaction of the patients.
\end{abstract}

Patients and Methods: Design: Study group: This study was conducted in Department of General Surgery Ain Shams University Hospitals, this is a prospective comparative study. The study included 40 women diagnosed with upper pole breast cancer. This study was done in Breast Surgery Unit, General Surgery Department at Ain Shams from July 1 st 2019 to July 1 2020 .

Sampling Method: Random controlled sampling study,after approval of the Ethical Committee, and informed consents were obtained from these patients they were enrolled in the study.

Results: Our study includes 40 female patients were randomly categorized into 2 groups. Group A included patients who had inferiority based wise pattern mammoplasty (20 patients), while Group B included patients who had batwing mammoplasty (20 patients).

Conclusion: In this study, we concluded that: Both techniques batwing mammoplasty and wise pattern therapeutic mammoplasty are valid options for upper pole breast tumors. Wise pattern therapeutic mammoplasty remains aesthetically superior; however, batwing mammoplasty is an easy, simple technique with acceptable results to patients.

Recommendations: From this study, we could recommend: The decision of which surgical approach to be used for the oncoplastic procedure is heavily based on patient and tumor characteristics; the pre-operative evaluation should include examination for degree of ptosis, overall skin quality, evidence

Correspondence to: Dr. Abd El-Rahman M. Essam, E-Mail: Abdothedoctor89@gmail.com of prior radiation, and overall breast size; successful oncoplastic procedure begins with selecting the appropriate operation for a given patient, which takes into account a patient's unique breast anatomy (e.g., breast shape and degree of ptosis) and good understanding of tumor location and extent, as well as appreciation of the patient's goals; Mastectomy with reconstruction may provide a more aesthetically pleasing result than breast conservation surgery in the small to moderatebreasted woman; larger breasted women have more options available for reconstruction, whether it is local tissue rearrangement, local or regional flaps, or reduction mammoplasty/mastopexy; Batwing mammoplasty procedure achieve optimal results (i.e., breast contour and nipple projection) in patients with larger breast volume and a mild to moderate degree of breast ptosis; more studies on a large scale should be performed to assess the results of batwing mammoplasty and wise pattern therapeutic mammoplasty regarding complication and cosmetic outcome for management of upper pole breast tumours.

Key Words: Breast conservation surgery $(B C S)$ - The nippleareola complex (NAC) - Oncoplastic surgery (OPS).

\section{Introduction}

SINCE the recorded time, breast has been a symbol for motherhood, fiminity, and sexuality, it has been portrayed throughout history in works of art symbolizing to all those aspects of woman's life. So breasts in female life are described as "life giving and life destroying" [1]

Breast cancer existed in ancient times and reference to this disease can be found dating back as $3000 \mathrm{BC}$, in an Egyptian papyrus [2] .

Breast cancer refers to cancer originating from breast tissue, most commonly from the inner lining of milk ducts or the lobules that supply the ducts with milk. It is a clonal disease; a single transformed cell the end results a series of somatic (acquired) or germ line mutations is able to express full malignant potential [3]. 
Breast cancer is first documented in the 7 th century B.C, it is the most common malignancy among women and the second leading cause of cancer women death. According to the WHO classification, breast tumors are divided into epithelial and mesenchymal types. Epithelial ones are more frequent and are further classified into noninvasive and invasive. According to their morphology, both of them are of ductal and lobular type. Amongst the invasive tumors, there are some special types which are different not only on a pure morphological basis, but also reflect different better or poorer prognosis [4].

The pathological types of breast cancer includes: Non-invasive "Carcinoma In Situ". (ductal, lobular), Invasive Carcinoma. (ductal, lobular, medullary, mucinous and tubular) and other special forms of invasive type (Inflammatory, Paget's disease) [5].

The establishment of modern radical surgery for breast cancer started with standard radical mastectomy, and then surgical procedures used for the breast cancer have been greatly changed from the standard radical mastectomy to breastconserving surgery. Today, the local control of breast cancer is the major objective of surgical treatment and is considered to be a part of systemic therapy. Breast-conserving surgery is the mainstay of treatment. However, about one-third of females with breast cancer still undergo mastectomy [6] .

Breast Conservation Surgery (BCS) combined with post-operative radiotherapy has become the preferred locoregional treatment for the majority of patients with early-stage breast cancer, with equivalent survival to that of mastectomy and improved body image and lifestyle scores. The success of BCS for breast cancer is based on the tenet of complete removal of the cancer with adequate surgical margins, while preserving the natural shape and appearance of the breast. Achieving both goals together in the same operation can be challenging, and BCS has not always produced good cosmetic results in all patients. One of the limiting factors is the amount of tissue removed, not only in terms of absolute volume but also in relation to tumor location and relative size of breast [7].

Oncoplastic Surgery (OPS) has emerged as a new approach to allow wide excision for BCS without compromising the natural shape of the breast. It is based upon integration of plastic surgery techniques for immediate breast reshaping after wide excision for breast cancer [7].
A number of factors influence the surgeon's choice of the surgical technique. Tumour size and location, as well as breast size and degree of ptosis, are some of the main factors considered in the decision making [8].

Upper pole tumours of the breast form a challenge to some extent to the oncoplastic surgeon, especially when considering the aesthetics of the breast. Scars in this area are very unsightly. In addition, less volume of tissue is available in the upper half of the breast to reconstruct the lumpectomy defect [9].

Aim of the work:

The aim of this study is to compare between batwing mammoplasty and inferiorly based wise pattern therapeutic mammoplasty in management of upper pole breast tumors regarding cosmetic results, oncological outcomes, rate of complications and degree of satisfaction of the patients.

\section{Patients and Methods}

\section{Study group:}

This study was conducted in Department of General Surgery Ain Shams University Hospitals, this is a prospective comparative study. The study included 40 women diagnosed with upper pole breast cancer. This study was done in Breast Surgery Unit, General Surgery Department at Ain Shams from July $1^{\text {st }} 2019$ to July $1^{\text {st }} 2020$.

\section{Sampling method:}

Random controlled sampling study,after approval of the ethical committee, and informed consents were obtained from these patients they were enrolled in the study.

\section{Inclusion criteria were:}

- Patients presented with upper pole breast malignant tumors (T1-2N0-1M0), which are defined as tumors occurring between 2 to 10 o'clock with distance between inferior margin of tumor and nipple areola complex not more than $5 \mathrm{~cm}$.

- Patients who completed their neoadjuvant treatment and did not miss follow-up.

\section{Exclusion criteria were:}

- Patients who are not candidate for breast conservative surgery as multicentric breast cancer and inflammatory breast cancer.

- Patients who missed their adjuvant treatment.

- Patients with metastatic breast cancer.

- Patients with auto-immune diseases. 


\section{Pre-operative and post-operative assessment:}

Patient underwent primary clinical assessment which include personal history, medical comorbidities, previous surgical history, history of pregnancy and lactation, current medications including contraceptive pills, family history of breast cancer then history of present illness and complaint.

General clinical examination of the patient then local examination of both breasts and axillae for proper clinical staging, assessment of tumor size, site and relation to nipple areola complex, skin and underlying muscle, axillary lymph node assessment.

Patient underwent routine pre-operative laboratory investigations including Complete blood count, liver and kidney function tests, coagulation profile, random blood sugar, virology markers (HBV, HCV) and tumor markers (CA 15.3).

Radiological investigations include bilateral sono-mammography for local assessment of tumor and axillary LNs. MRI breast done in patients with dense breast tissues in sono-mammography to assess tumor extension and exclude presence of multicentric or multifocal disease, CT chest and abdomen with contrast as metastatic work up, bone scan done in patients complaining from bone aches to exclude bone metastases, CT brain also done in case of any neurological manifestations.

All cases underwent true-cut needle biopsy from the tumor for pathological diagnosis.

After all investigations are done, with no data consider distant metastases or locally advanced disease, all cases discussed by multidisciplinary team to decide the patient's plan of management.

Appropriate counselling of the patients regarding the procedure and contralateral mammoplasty with the same technique, and patients' consent was taken.

The patient population were randomly categorized into 2 groups. Group A included patients who had inferiority based wise pattern mammoplasty (20 patients). While Group B included patients who had batwing mammoplasty (20 patients).

\section{Pre-operative preparation:}

All cases admitted one day before surgery, fasting for 8 hours before time of operation, received pre-operative prophylactic antibiotic, drawing of incision site before surgery.

\section{Operative procedures:}

Batwing mammoplasty procedure includes two similar semi-circular incision with angled (wings) on each side of the areola, the two semicircular wings are positioned so they can be re-approximated to each other at wound closure. Removal of skin wings allow the two semicircles to shift together.

Inferiorly based wise pattern therapeutic mammoplasty based on inferior pedicle, removes tissue from around the pedicle medially, superiorly and laterally, removes the skin below medial and lateral flaps, including de-epithelialized inferior pedicle and reshape the skin around the inferior pedicle to shape the breast.

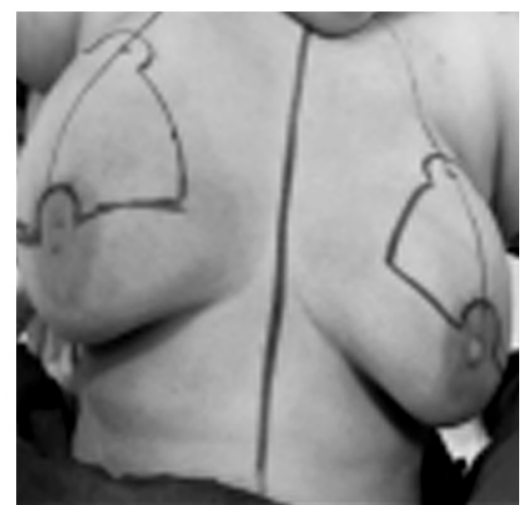

Fig. (1): Pre-operative marking of incision. 

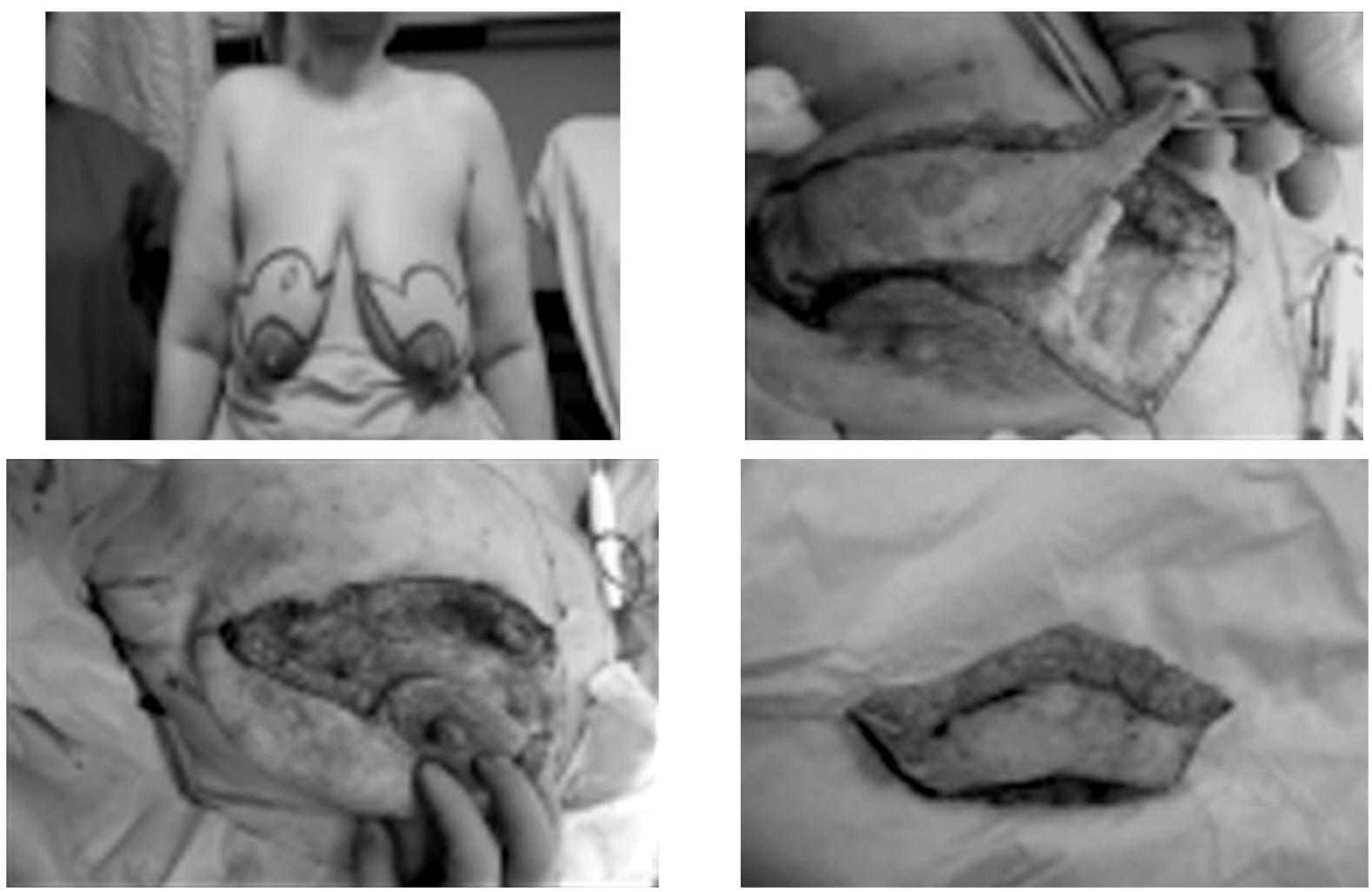

Fig. (2): The operative steps of batwing mammoplasty.
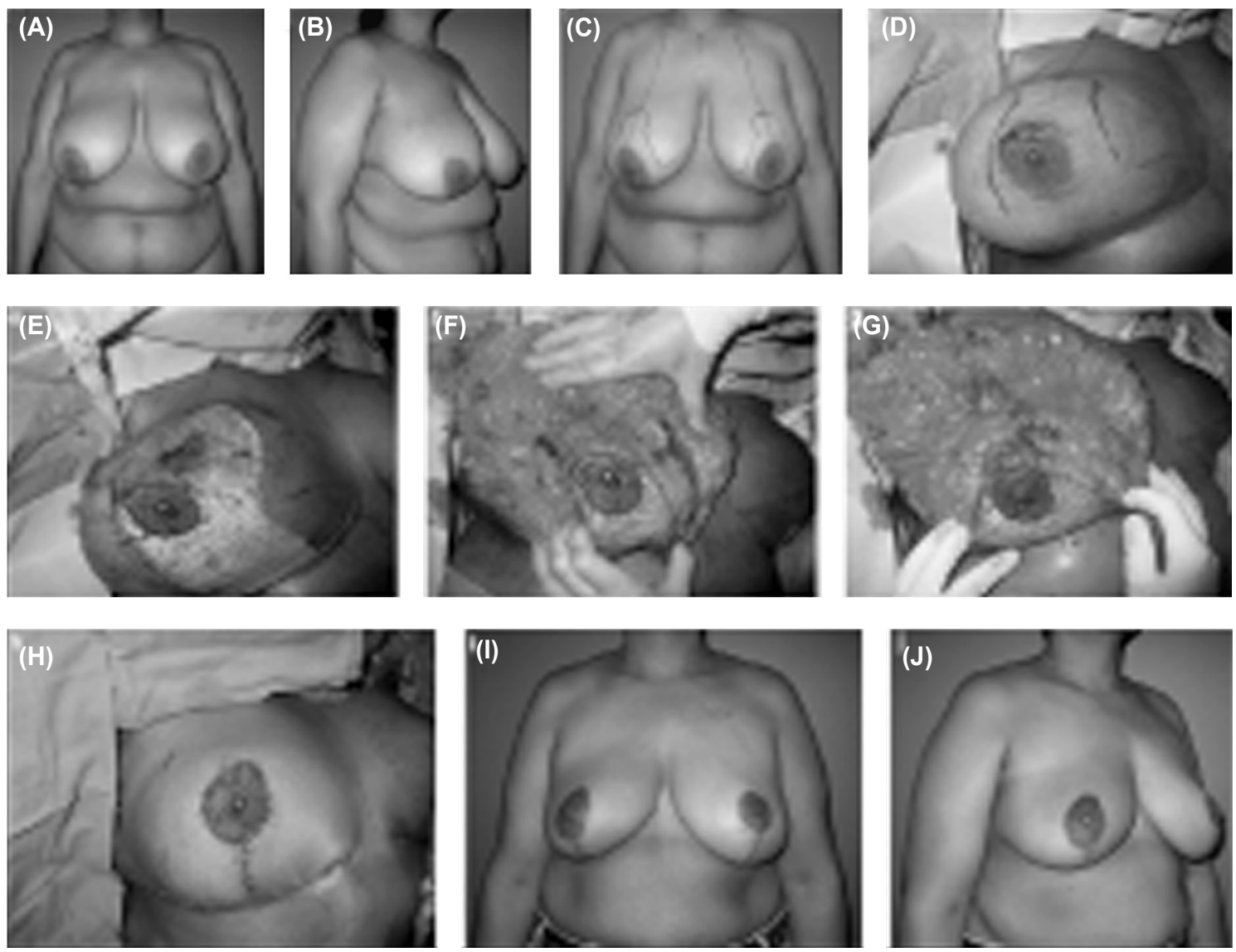

Fig. (3): The operative steps of inferiorly based wise pattern therapeutic mammoplasty. 
Specimens sent for frozen section immediately after wide local excision to confirm that resection margins are free of tumor and to assess presence of insitu component. In both techniques, double limb suction drain applied at bed of tumor and axilla.

\section{Post-operative assessment:}

Patient stayed at hospital from 2-3 days then discharged with drains, regular follow-up in outpatient clinic was done after 1 week then 2 weeks post-operative for wound care and monitoring of drains output and assessment of any complications. Drains removed when their output is less than $50 \mathrm{cc} / 24 \mathrm{hrs}$ and all patients referred to start adjuvant therapy after complete wounds healing, removal of sutures and drains.

Post-operative outpatient visits for regular follow-up and monitoring of outcome was every week at the first month after surgery, then a monthly visit for 6 months. With monthly follow-up visit at surgery clinic to assess the surgical outcome, also, clinical, radiological, oncological follow-up was performed.

After 6 months of regular follow-up with the patients we asked them to fill five-scale questionnaire evaluating their own cosmetic outcome graded as (5. excellent, 4. good, 3. fair, 2. poor, 1. very poor).

\section{Statistical analysis:}

The data collected were tabulated and analyzed by SPSS (statistical package for social science) version 20.0 on IBM compatible computer.

Two types of statistics were done including descriptive statisticse.g., percentage (\%), mean, median, range, and standard deviation (SD) and analytic statisticse.g.: $p$-value, $\mathrm{X} 2$.

$p$-value varies from 0 to 1 and is defined as follows. A small $p$-value (typically $<0.05$ ) implies strong evidence against the null hypothesis, so you dismiss the null hypothesis. But, a large $p$-value $(>0.05)$ indicates weak evidence against the null hypothesis; the null hypothesis is not rejected while $p$-values that are very close to the cutoff $(0.05)$ are considered marginal (may go either way).

\section{Results}

Our study includes 40 female patients were randomly categorized into 2 groups.

Group A included patients who had inferiority based wise pattern mammoplasty (20 patients), while Group B included patients who had batwing mammoplasty (20 patients).

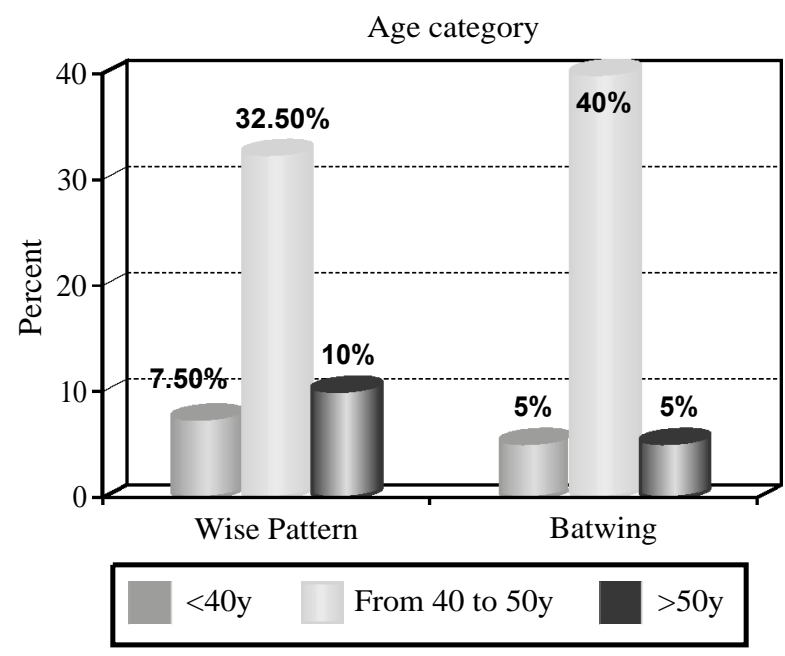

Fig. (1): This figure shows age of patients' population.

Table (1): Age of the patients.

\begin{tabular}{|c|c|c|c|c|c|c|c|c|}
\hline \multirow{2}{*}{$\begin{array}{l}\text { Mamoplastic } \\
\text { surgery }\end{array}$} & \multicolumn{5}{|c|}{ Age } & \multirow{2}{*}{$\begin{array}{l}\text { Mean } \\
\text { rank }\end{array}$} & \multirow{2}{*}{$\begin{array}{l}\text { Mann- } \\
\text { Whitney } \\
\text { U-Test }\end{array}$} & \multirow{2}{*}{$\begin{array}{c}p- \\
\text { value }\end{array}$} \\
\hline & $\begin{array}{c}\text { Mean } \\
\text { (range) }\end{array}$ & $\begin{array}{c}<40 y \\
\text { No }(\%)\end{array}$ & $\begin{array}{c}\text { from } 40 \text { to } 50 \mathrm{y} \\
\text { No }(\%)\end{array}$ & $\begin{array}{c}>50 y \\
\text { No }(\%)\end{array}$ & $\begin{array}{c}\text { Total } \\
\text { No }(\%)\end{array}$ & & & \\
\hline Wise pattern & $45.7(36-57)$ & $3(15 \%)$ & $13(65 \%)$ & $4(20 \%)$ & $20(100 \%)$ & 20.95 & 191 & 0.756 \\
\hline Batwing & $44.6(38-52)$ & $2(10 \%)$ & $16(80 \%)$ & $2(10 \%)$ & $20(100 \%)$ & 20.05 & & \\
\hline
\end{tabular}

This table shows: The mean age of participants in wise pattern operation was 45.7 years with a range of 36-57 years. While in batwing operation participants had a mean age of 44.6 years with a range of 38-52 years, there is no significant statistical difference between different age groups. 
Table (2): Medical history and comorbidities of the patients.

\begin{tabular}{|c|c|c|c|}
\hline Group & No $(\%)$ & Fisher's Exact Test & $p$-value \\
\hline \multicolumn{4}{|l|}{ Wise pattern: } \\
\hline Normal & $17(85 \%)$ & 1.59 & $p=1$ \\
\hline Asthmatic & $1 \quad(5 \%)$ & & \\
\hline DM & $1 \quad(5 \%)$ & & \\
\hline HTN & $1 \quad(5 \%)$ & & \\
\hline Total & $20(100 \%)$ & & \\
\hline \multicolumn{4}{|l|}{ Batwing: } \\
\hline Normal & $17(85 \%)$ & & \\
\hline DM & $2(10 \%)$ & & \\
\hline HTN & $1 \quad(5 \%)$ & & \\
\hline Total & $20(100 \%)$ & & \\
\hline
\end{tabular}

This table shows: The total number of patients was (40) patients divided in two groups each group had twenty patients. One patient inwise pattern group had hypertension, one had type II diabetes and one asthmatic. On the other hand, one patient in batwing group had hypertension and two had type II diabetes. No significant statistical difference between different groups with medical comorbidities.

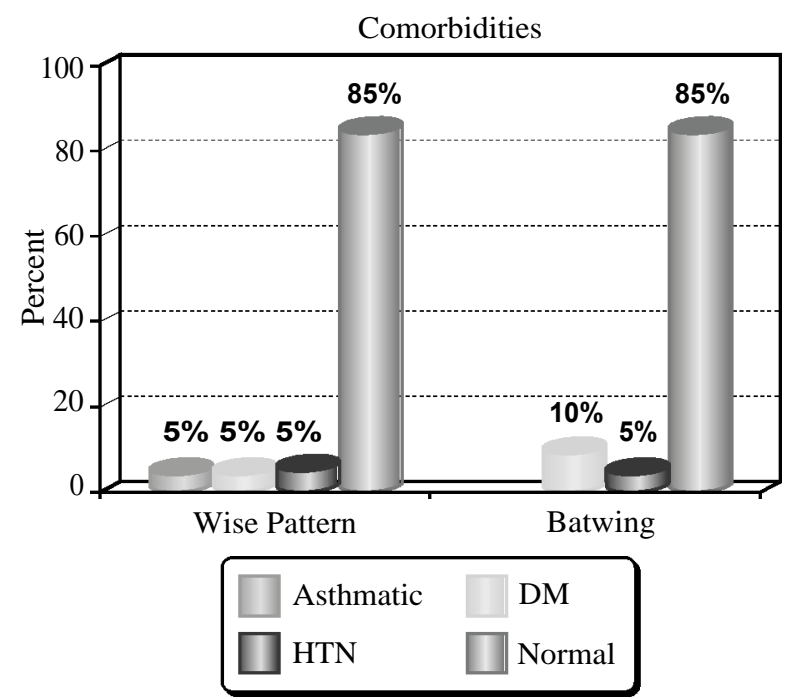

Fig. (2): This figure shows comorbidity distribution of the patients.

Table (3): Degree of breast ptosis of the patients.

\begin{tabular}{lcc}
\hline & $\begin{array}{c}\text { Wise pattern } \\
\text { No }(\%)\end{array}$ & $\begin{array}{c}\text { Batwing } \\
\text { No }(\%)\end{array}$ \\
\hline $\begin{array}{l}\text { Degree of breast ptosis: } \\
\text { Type 1 }\end{array}$ & $2(10 \%)$ & $1(5 \%)$ \\
Type 2 & $14(70 \%)$ & $15(75 \%)$ \\
Type 3 & $4(20 \%)$ & $4 \quad(20 \%)$ \\
Fisher's Exact Test & \multicolumn{2}{c}{0.512} \\
& \multicolumn{3}{c}{$p$-value $=1$} \\
\hline
\end{tabular}

This table shows: In wise pattern group there were 2 patients with type (1) ptosis, (14) patients with type 2 ptosis and (4) patients with type 3 ptosis while in Batwing group there were (1) patient with type 1 ptosis, (15) patients with type 2 ptosis and (4) patients with type 3 ptosis. No significant statistical difference between different groups of breast ptosis.

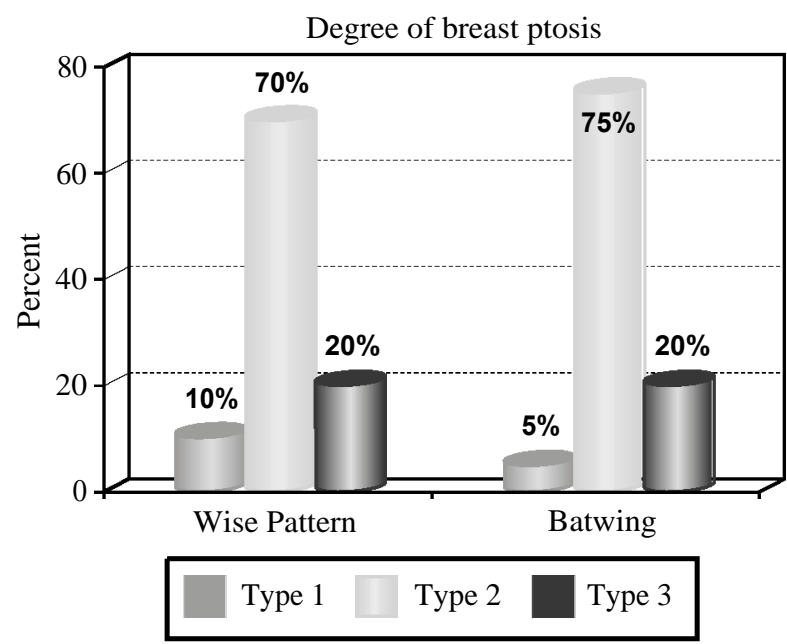

Fig. (3): This figure shows pre-operative assessment of breast ptosis

Table (4): Post-operative pathology assessment.

\begin{tabular}{lll}
\hline Pathology & $\begin{array}{c}\text { Wise pattern } \\
\text { No }(\%)\end{array}$ & $\begin{array}{l}\text { Batwing } \\
\text { No }(\%)\end{array}$ \\
\hline IDC & $18(90 \%)$ & $19(95 \%)$ \\
ILC & $2(10 \%)$ & $1(5 \%)$ \\
\hline
\end{tabular}

This table shows: In Wise Pattern group 2 patients had ILC while the others had IDC. In Batwing group one patient had ILC while the rest of the patients had IDC. All cases showed free margins of resection that was confirmed intraoperative by frozen section.

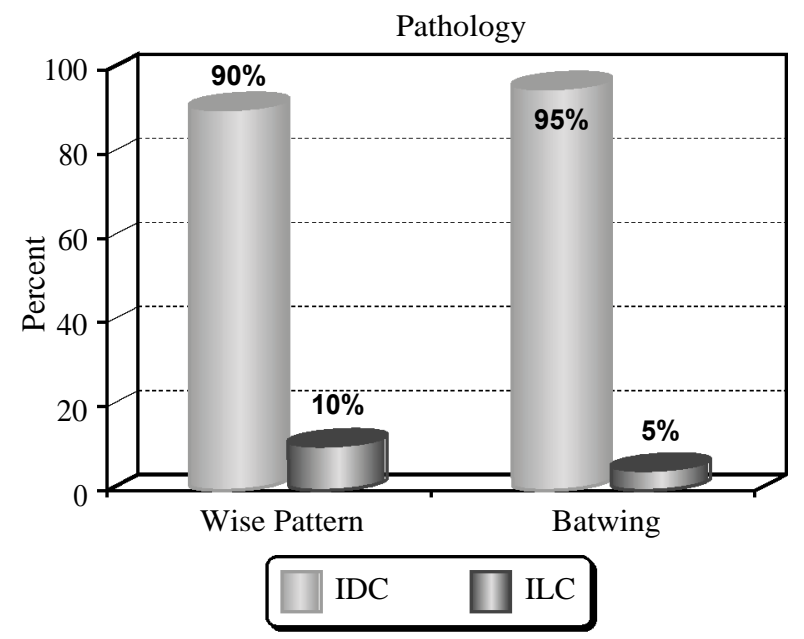

Fig. (4): This figure shows the type of pathology. 
Table (5): Tumor staging.

\begin{tabular}{lcc}
\hline & $\begin{array}{c}\text { Wise pattern } \\
\text { No }(\%)\end{array}$ & $\begin{array}{c}\text { Batwing } \\
\text { No }(\%)\end{array}$ \\
\hline Staging: & $2(10 \%)$ & $1(5 \%)$ \\
T1N0 & $2(10 \%)$ & $3(15 \%)$ \\
T1N1 & $5(25 \%)$ & $3(15 \%)$ \\
T2N0 & $11(55 \%)$ & $13(65 \%)$ \\
T2N1 & \multicolumn{2}{c}{2.028} \\
Fisher's Exact Test & \multicolumn{2}{c}{$p$-value $=0.675$} \\
\hline
\end{tabular}

This table shows: In wise Pattern group there were (11) patients in T2N1 stage, (5) patients in T2N0 stage and (2) patients in T1N1 stage and (2) patients in T $1 \mathrm{~N} 0$ stage, while in batwing group there were (13) patients in T2N1 stage, (3) patient in T2N0 stage, (3) patients in T1N1 stage and (1) patients in T1N0 stage. No significant statistical difference between different groups of tumor staging.

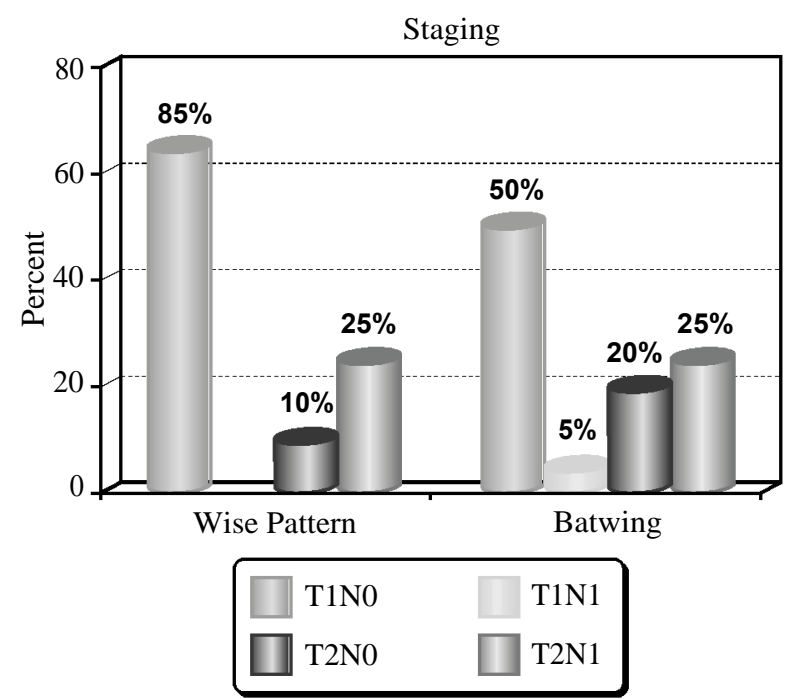

Fig. (5): This figure shows the assessment of TNM staging in both groups.

Simple Boxplot of time of operation by Mamoplastic surgery

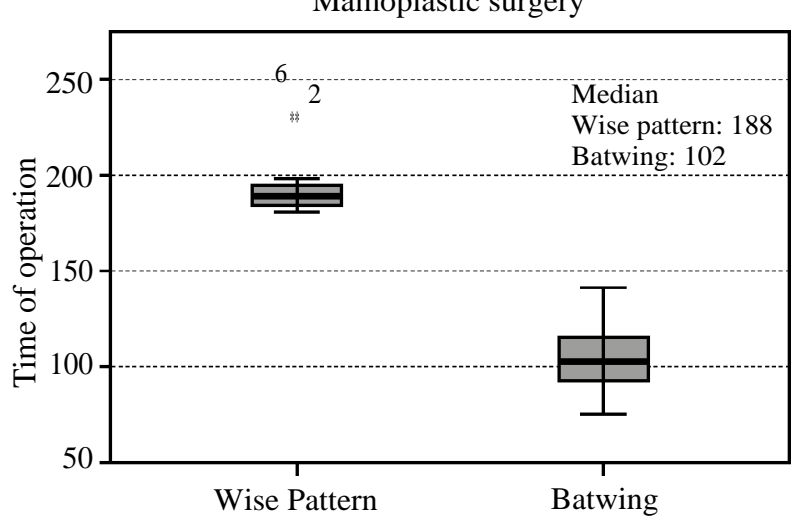

Fig. (6): This figure shows the difference between time of both operation.
Table (6): Time of operation.

\begin{tabular}{lllll}
\hline $\begin{array}{l}\text { Time of } \\
\text { operation }\end{array}$ & Mean \pm SD & \multicolumn{1}{c}{$\begin{array}{c}\text { Median } \\
\text { (range) }\end{array}$} & $\chi^{2}$ & $\begin{array}{c}p \text { - } \\
\text { value }\end{array}$ \\
\hline $\begin{array}{l}\text { Wise pattern } \\
\text { Batwing }\end{array}$ & $\begin{array}{l}192.3 \pm 14.72 \\
104 \pm 18.25\end{array}$ & $188(180-235)$ & 40 & 0.029 \\
\hline
\end{tabular}

This table shows: There was significant difference in the time of operation between wise pattern and batwing groups where the mean operation time in batwing (104 $\pm 18.25 \mathrm{~min})$ was shorter than mean operation time in wise pattern $(192.3 \pm 14.72 \mathrm{~min})$.

Table (7): Weight of specimen obtained from the patients.

\begin{tabular}{lllll}
\hline $\begin{array}{l}\text { Weight of } \\
\text { specimen }\end{array}$ & Mean \pm SD & \multicolumn{1}{c}{$\begin{array}{l}\text { Median } \\
\text { (range) }\end{array}$} & $\chi^{2}$ & $\begin{array}{c}p \text { - } \\
\text { value }\end{array}$ \\
\hline $\begin{array}{l}\text { Wise pattern } \\
\text { Batwing }\end{array}$ & $\begin{array}{l}630.3 \pm 81.29 \\
725 \pm 96.78\end{array}$ & $\begin{array}{l}625.5(504-791) \\
730.5(573-859)\end{array}$ & 38 & 0.378 \\
\hline
\end{tabular}

This table shows: The mean weight of specimen in wise pattern was $(630.3 \pm 81.29 \mathrm{gm})$ which is less than then mean weight of specimen in batwing $(725 \pm 96.78 \mathrm{gm})$. With no significant statistical difference between both groups.

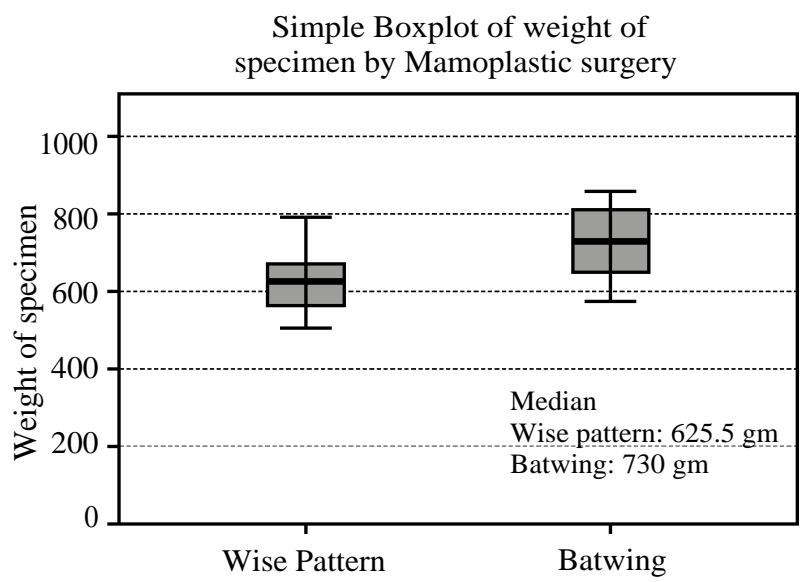

Fig. (7): This figure shows the difference between breast tissue excised in both operation.

Table (8): Hospital stay of the patients of both groups.

\begin{tabular}{|c|c|c|c|c|}
\hline $\begin{array}{l}\text { Hospital } \\
\text { stay }\end{array}$ & Mean \pm SD & $\begin{array}{c}\text { Median } \\
\text { (range) }\end{array}$ & $\chi^{2}$ & $\begin{array}{c}p- \\
\text { value }\end{array}$ \\
\hline Wise pattern & $2.4 \pm 0.82$ & $2(2-5)$ & 1.33 & 0.723 \\
\hline Batwing & $2.2 \pm 0.52$ & $2(2-4)$ & & \\
\hline
\end{tabular}

This table shows: No significant statistical difference between different groups regarding postoperative hospital stay, with mean hospital stay in wise pattern ( $2.4 \pm 0.82$ day), while the mean hospital stay after batwing was $(2.2 \pm 0.52)$. 


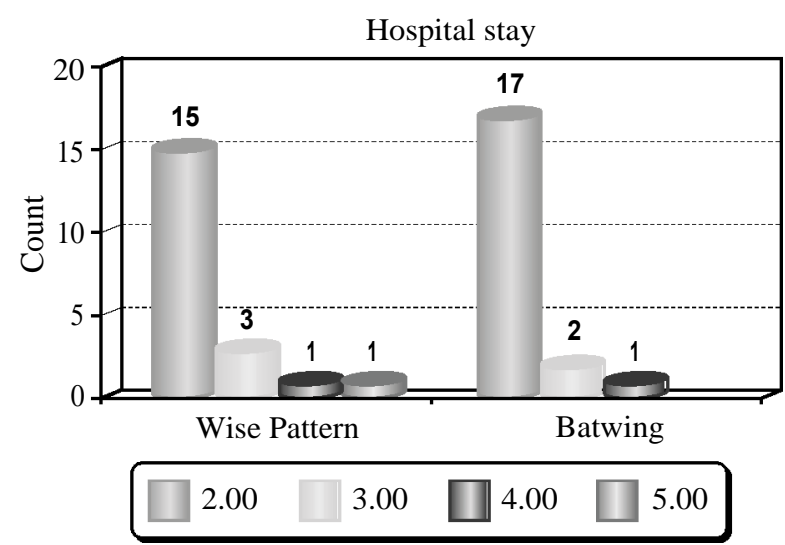

Fig. (8): This figure shows the difference between hospital stay in both operations.

Table (9): Post-operative assessment of the breast wound.

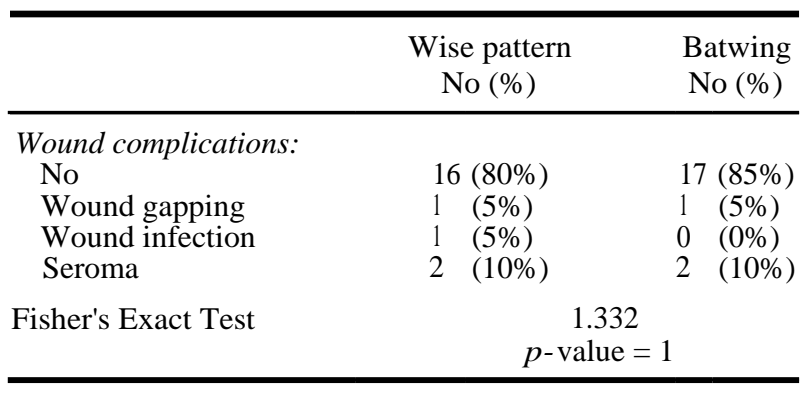

This table shows:

- One patient from each group presented with gapped wound at the 2-week post-surgery, the patient in batwing group managed with repeated dressing, the wise pattern patient needed secondary sutures.

- There was one patient in wise pattern group had an infected wound, managed by systemic and topical antibiotics with local wound care and regular dressing.

- Two patients in each group suffered from seroma after operation that were managed conservatively by medical treatment. No significant statistical difference between different groups with wound complications.

Table (10): Post-operative assessment of the nipple and areola.

\begin{tabular}{|c|c|c|}
\hline & $\begin{array}{c}\text { Wise pattern } \\
\text { No }(\%)\end{array}$ & $\begin{array}{l}\text { Batwing } \\
\text { No }(\%)\end{array}$ \\
\hline $\begin{array}{l}\text { Nipple and areola } \\
\text { Intact } \\
\text { Sloughed areola }\end{array}$ & $\begin{array}{l}18(90 \%) \\
2 \quad(10 \%)\end{array}$ & $\begin{array}{l}20(100 \%) \\
0 \quad(0 \%)\end{array}$ \\
\hline Fisher's Exact Test & \multicolumn{2}{|c|}{$\begin{array}{c}2.105 \\
p \text {-value }=0.487\end{array}$} \\
\hline $\begin{array}{l}\text { Nipple and areola } \\
\text { Intact } \\
\text { Loss }\end{array}$ & $\begin{array}{ll}17 & (85 \%) \\
3 & (15 \%)\end{array}$ & $\begin{array}{l}20(100 \%) \\
0 \quad(0 \%)\end{array}$ \\
\hline Fisher's Exact Test & \multicolumn{2}{|c|}{$\begin{array}{c}3.243 \\
p \text {-value }=0.231\end{array}$} \\
\hline
\end{tabular}

This table shows: There was no significant difference between wise Pattern group and batwing group regarding nipple and areola vasculature and sensation. Two patients in wise Pattern group (10\%) reported sloughing of areola while no cases were reported in batwing group. Two patients in wise Pattern group $(10 \%)$ reported loss of sensation of nipple and areola complex while no cases in batwing group. No significant statistical difference between different groups with nipple and areola complications.

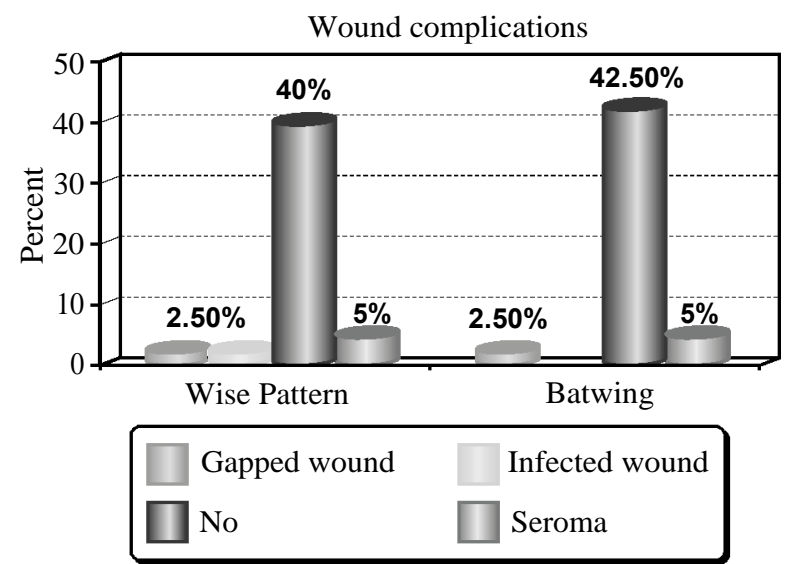

Fig. (9): This figure shows the assessment of wound complication in both groups.

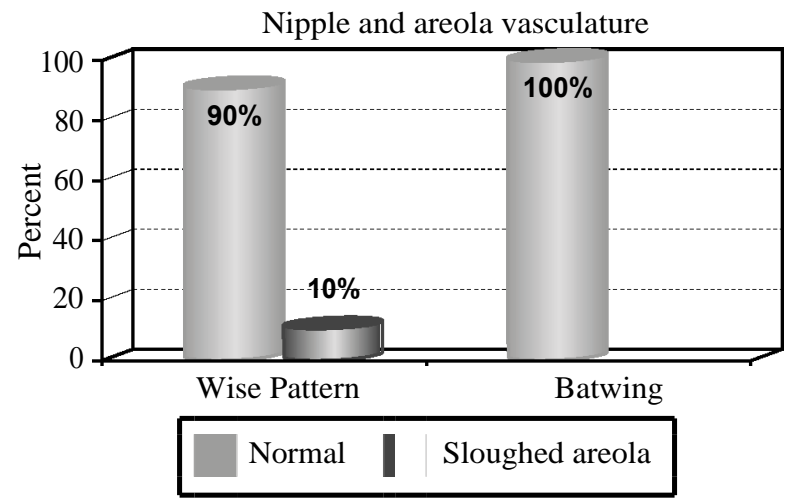

Fig. (10): This figure shows the assessment of nipple and areola vasculature in both groups.

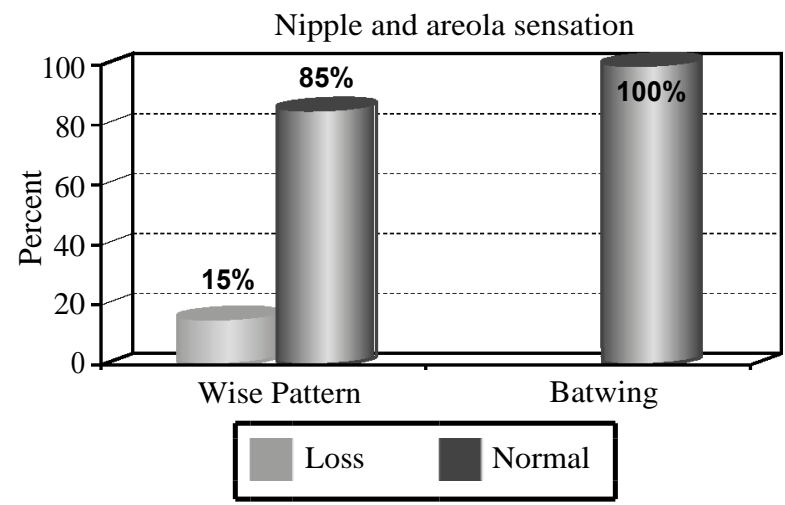

Fig. (11): This figure shows the assessment of nipple and areola sensation in both groups. 
Table (11): Assessment of cosmetic output for both operations by the patients.

\begin{tabular}{|c|c|c|c|c|c|c|c|c|}
\hline & $\begin{array}{l}\text { Very poor } \\
\text { No }(\%)\end{array}$ & $\begin{array}{c}\text { Poor } \\
\text { No }(\%)\end{array}$ & $\begin{array}{c}\text { Fair } \\
\text { No }(\%)\end{array}$ & $\begin{array}{c}\text { Good } \\
\text { No }(\%)\end{array}$ & $\begin{array}{l}\text { Excellent } \\
\text { No }(\%)\end{array}$ & $\begin{array}{l}\text { Mean } \\
\text { Rank }\end{array}$ & $\begin{array}{c}\text { Mann-Whitney } \\
\text { Test }\end{array}$ & $\begin{array}{c}p- \\
\text { value }\end{array}$ \\
\hline $\begin{array}{l}\text { Shape: } \\
\text { Wise pattern } \\
\text { Batwing }\end{array}$ & $\begin{array}{l}0(0 \%) \\
0(0 \%)\end{array}$ & $\begin{array}{l}2(10 \%) \\
3(15 \%)\end{array}$ & $\begin{array}{l}6(30 \%) \\
6(30 \%)\end{array}$ & $\begin{array}{l}9(45 \%) \\
10(50 \%)\end{array}$ & $\begin{array}{l}3(15 \%) \\
1(5 \%)\end{array}$ & $\begin{array}{l}20.15 \\
20.85\end{array}$ & 193 & 0.84 \\
\hline $\begin{array}{l}\text { Volume: } \\
\text { Wise pattern } \\
\text { Batwing }\end{array}$ & $\begin{array}{l}0(0 \%) \\
1(5 \%)\end{array}$ & $\begin{array}{l}0(0 \%) \\
3(15 \%)\end{array}$ & $\begin{array}{l}7(35 \%) \\
5(25 \%)\end{array}$ & $\begin{array}{l}10(50 \%) \\
10(50 \%)\end{array}$ & $\begin{array}{l}3(15 \%) \\
1(5 \%)\end{array}$ & $\begin{array}{l}22.70 \\
18.30\end{array}$ & 156 & 0.196 \\
\hline $\begin{array}{l}\text { Ptosis: } \\
\text { Wise pattern } \\
\text { Batwing }\end{array}$ & $\begin{array}{l}0(0 \%) \\
0(0 \%)\end{array}$ & $\begin{array}{l}0(0 \%) \\
2(10 \%)\end{array}$ & $\begin{array}{l}5(25 \%) \\
9(45 \%)\end{array}$ & $\begin{array}{l}9(45 \%) \\
6(30 \%)\end{array}$ & $\begin{array}{l}6(30 \%) \\
3(15 \%)\end{array}$ & $\begin{array}{l}23.98 \\
17.02\end{array}$ & 130.5 & 0.047 \\
\hline $\begin{array}{l}\text { Projection: } \\
\text { Wise pattern } \\
\text { Batwing }\end{array}$ & $\begin{array}{l}0(0 \%) \\
1(5 \%)\end{array}$ & $\begin{array}{l}0(0 \%) \\
4(20 \%)\end{array}$ & $\begin{array}{l}4(20 \%) \\
10(50 \%)\end{array}$ & $\begin{array}{l}9(45 \%) \\
5(25 \%)\end{array}$ & $\begin{array}{l}7(35 \%) \\
0(0 \%)\end{array}$ & $\begin{array}{l}27.38 \\
13.63\end{array}$ & 62.5 & $<0.001$ \\
\hline $\begin{array}{l}\text { Symmetry: } \\
\text { Wise pattern } \\
\text { Batwing }\end{array}$ & $\begin{array}{l}0(0 \%) \\
1(5 \%)\end{array}$ & $\begin{array}{l}2(10 \%) \\
2(10 \%)\end{array}$ & $\begin{array}{l}4(20 \%) \\
6(30 \%)\end{array}$ & $\begin{array}{l}9(45 \%) \\
11(55 \%)\end{array}$ & $\begin{array}{l}5(25 \%) \\
0(0 \%)\end{array}$ & $\begin{array}{l}22.78 \\
18.23\end{array}$ & 154.5 & 0.185 \\
\hline $\begin{array}{l}\text { Scar visibility: } \\
\text { Wise pattern } \\
\text { Batwing }\end{array}$ & $\begin{array}{l}0(0 \%) \\
1(5 \%)\end{array}$ & $\begin{array}{l}3(15 \%) \\
2(10 \%)\end{array}$ & $\begin{array}{l}7(35 \%) \\
5(25 \%)\end{array}$ & $\begin{array}{l}9(45 \%) \\
9(45 \%)\end{array}$ & $\begin{array}{l}1(5 \%) \\
3(15 \%)\end{array}$ & $\begin{array}{l}25.58 \\
15.43\end{array}$ & 98.5 & 0.003 \\
\hline $\begin{array}{l}\text { Overall satisfaction: } \\
\text { Wise pattern } \\
\text { Batwing }\end{array}$ & $\begin{array}{l}0(0 \%) \\
0(0 \%)\end{array}$ & $\begin{array}{l}3(15 \%) \\
1(5 \%)\end{array}$ & $\begin{array}{l}8(40 \%) \\
3(15 \%)\end{array}$ & $\begin{array}{l}7(35 \%) \\
11(55 \%)\end{array}$ & $\begin{array}{l}2(10 \%) \\
5(25 \%)\end{array}$ & $\begin{array}{l}23.95 \\
17.05\end{array}$ & 131 & 0.048 \\
\hline
\end{tabular}

This table shows:

- As regard to the patient assessment of cosmetic output, they were asked to fill a five-scale questionnaire evaluating their own cosmetic outcome graded as (5. excellent, 4. good, 3. fair, 2. poor, 1 . very poor) after 6 months of regular followup after performing their operations.

- There were significant differences between the two operations in scar visibility, breast projection and overall satisfaction in favor of wise pattern operation.

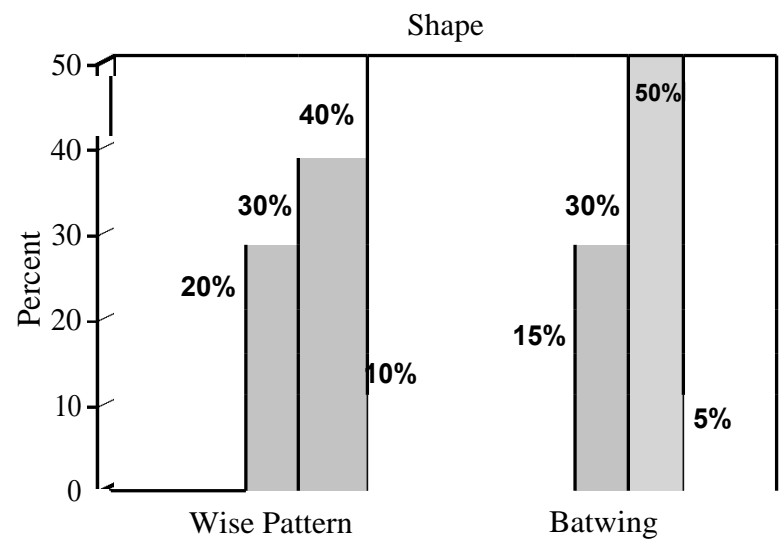

Mamoplastic Surgery

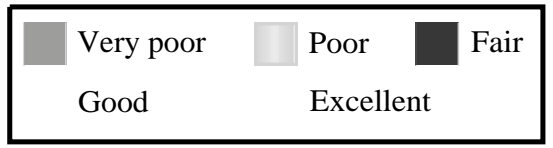

Fig. (12): This figure shows the post-operative assessment of the shape of the breast for both operations.

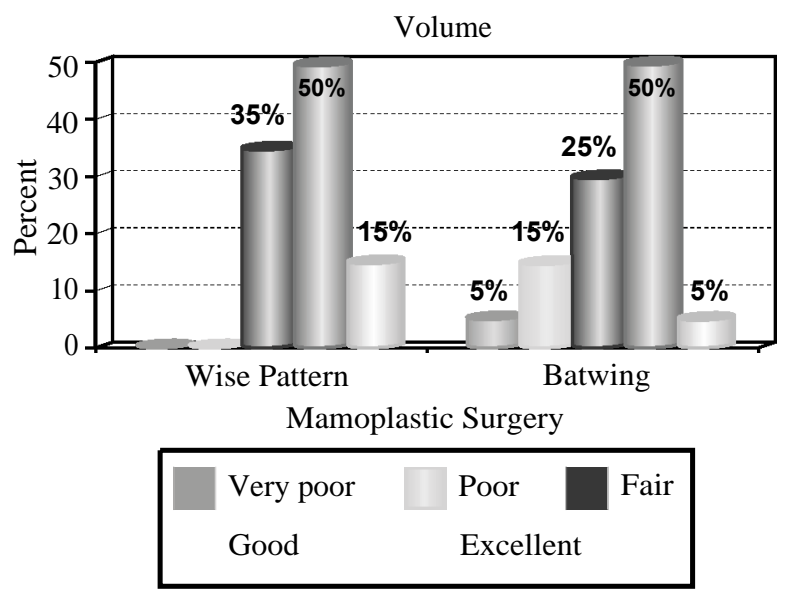

Fig. (13): This figure shows the post-operative assessment of the volume of the breast for both operations.

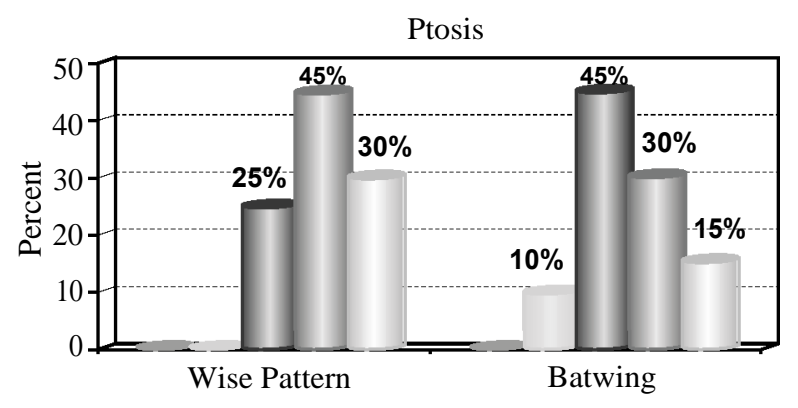

Mamoplastic Surgery

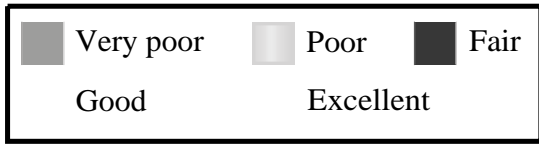

Fig. (14): This figure shows the post-operative assessment of ptosis of the breast for both operations. 


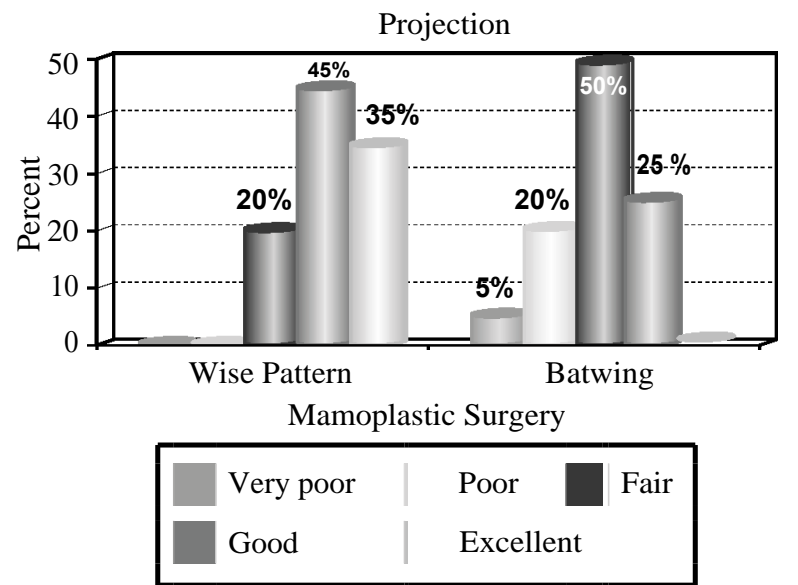

Fig. (15): This figure shows the post-operative assessment of breast projection for both operation.

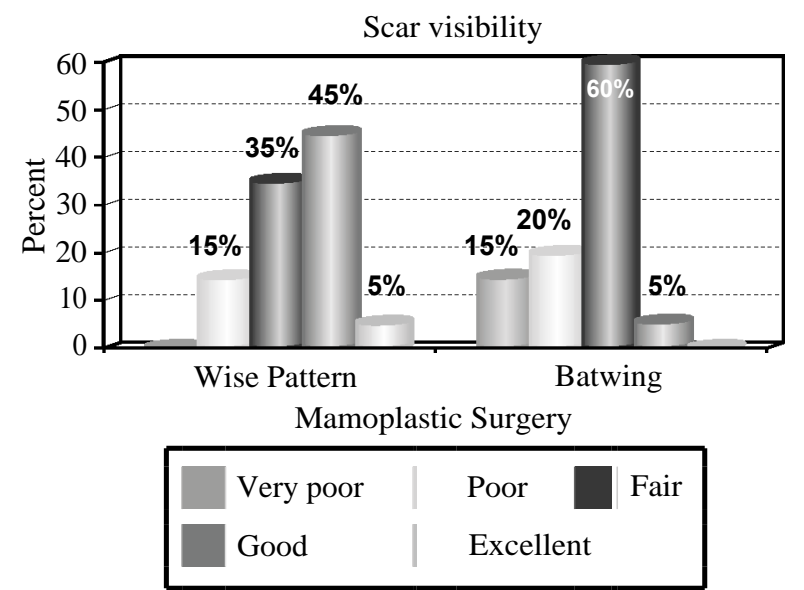

Fig. (17): This figure shows the post-operative assessment of scar visibility for both operations.

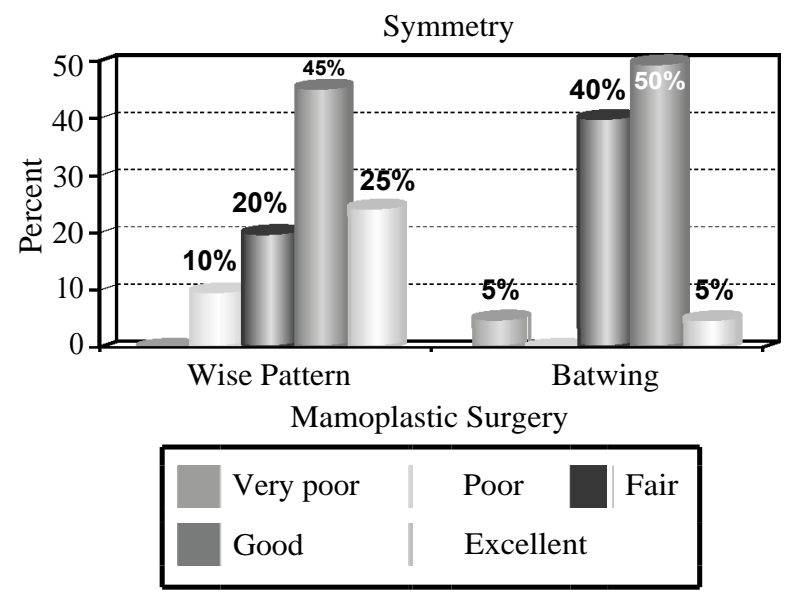

Fig. (16): This figure shows the post-operative assessment of breast symmetry for both operations.

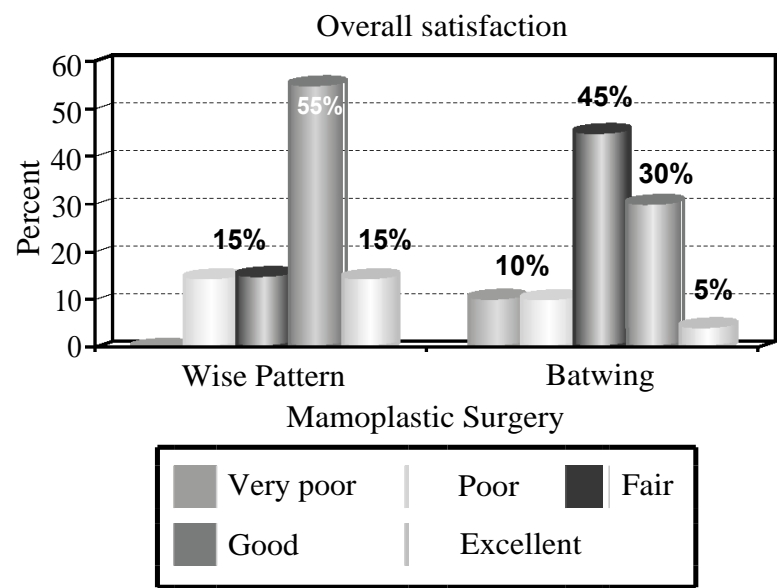

Fig. (18): This figure shows the post-operative assessment of overall satisfaction of the patient for both operations.

Table (12): Assessment of cosmetic output for both operation by the physician.

\begin{tabular}{|c|c|c|c|c|c|c|c|c|}
\hline & $\begin{array}{l}\text { Very poor } \\
\text { No }(\%)\end{array}$ & $\begin{array}{c}\text { Poor } \\
\text { No }(\%)\end{array}$ & $\begin{array}{c}\text { Fair } \\
\text { No }(\%)\end{array}$ & $\begin{array}{c}\text { Good } \\
\text { No }(\%)\end{array}$ & $\begin{array}{l}\text { Excellent } \\
\text { No }(\%)\end{array}$ & $\begin{array}{l}\text { Mean } \\
\text { Rank }\end{array}$ & $\begin{array}{c}\text { Mann-Whitney } \\
\text { Test }\end{array}$ & $\begin{array}{c}p- \\
\text { value }\end{array}$ \\
\hline $\begin{array}{l}\text { Shape: } \\
\text { Wise pattern } \\
\text { Batwing }\end{array}$ & $\begin{array}{l}0(0 \%) \\
0(0 \%)\end{array}$ & $\begin{array}{l}1(5 \%) \\
3(15 \%)\end{array}$ & $\begin{array}{l}4(20 \%) \\
7(35 \%)\end{array}$ & $\begin{array}{l}11(55 \%) \\
8 \quad(40 \%)\end{array}$ & $\begin{array}{l}4(20 \%) \\
2(10 \%)\end{array}$ & $\begin{array}{l}21.88 \\
19.13\end{array}$ & 172.5 & 0.425 \\
\hline $\begin{array}{l}\text { Volume: } \\
\text { Wise pattern } \\
\text { Batwing }\end{array}$ & $\begin{array}{l}0(0 \%) \\
0(0 \%)\end{array}$ & $\begin{array}{l}0(0 \%) \\
4(20 \%)\end{array}$ & $\begin{array}{l}6(30 \%) \\
7(35 \%)\end{array}$ & $\begin{array}{l}12(60 \%) \\
8 \quad(40 \%)\end{array}$ & $\begin{array}{l}2(10 \%) \\
1(5 \%)\end{array}$ & $\begin{array}{l}23.7 \\
17.3\end{array}$ & 136 & 0.059 \\
\hline $\begin{array}{l}\text { Ptosis: } \\
\text { Wise pattern } \\
\text { Batwing }\end{array}$ & $\begin{array}{l}0(0 \%) \\
1(5 \%)\end{array}$ & $\begin{array}{l}0(0 \%) \\
4(20 \%)\end{array}$ & $\begin{array}{l}7 \quad(35 \%) \\
10(50 \%)\end{array}$ & $\begin{array}{l}10(50 \%) \\
4(20 \%)\end{array}$ & $\begin{array}{l}3(60 \%) \\
1(5 \%)\end{array}$ & $\begin{array}{l}23.75 \\
17.25\end{array}$ & 135 & 0.063 \\
\hline $\begin{array}{l}\text { Projection: } \\
\text { Wise pattern } \\
\text { Batwing }\end{array}$ & $\begin{array}{l}0(0 \%) \\
1(5 \%)\end{array}$ & $\begin{array}{l}0(0 \%) \\
6(30 \%)\end{array}$ & $\begin{array}{l}6(30 \%) \\
8(40 \%)\end{array}$ & $\begin{array}{l}9(45 \%) \\
5(25 \%)\end{array}$ & $\begin{array}{l}5(25 \%) \\
0(0 \%)\end{array}$ & $\begin{array}{l}26.88 \\
14.33\end{array}$ & 76.5 & $<0.001$ \\
\hline $\begin{array}{l}\text { Symmetry: } \\
\text { Wise pattern } \\
\text { Batwing }\end{array}$ & $\begin{array}{l}0(0 \%) \\
1(5 \%)\end{array}$ & $\begin{array}{l}0(0 \%) \\
3(15 \%)\end{array}$ & $\begin{array}{l}6(30 \%) \\
8(40 \%)\end{array}$ & $\begin{array}{l}8(40 \%) \\
8(40 \%)\end{array}$ & $\begin{array}{l}6(30 \%) \\
1(5 \%)\end{array}$ & $\begin{array}{l}22.6 \\
18.4\end{array}$ & 158 & 0.224 \\
\hline $\begin{array}{l}\text { Scar visibility: } \\
\text { Wise pattern } \\
\text { Batwing }\end{array}$ & $\begin{array}{l}0(0 \%) \\
3(15 \%)\end{array}$ & $\begin{array}{l}1(5 \%) \\
4(20 \%)\end{array}$ & $\begin{array}{l}5(25 \%) \\
9(45 \%)\end{array}$ & $\begin{array}{l}8(40 \%) \\
3(15 \%)\end{array}$ & $\begin{array}{l}6(30 \%) \\
1(5 \%)\end{array}$ & $\begin{array}{l}26.18 \\
14.83\end{array}$ & 86.5 & 0.001 \\
\hline $\begin{array}{l}\text { Overall satisfaction: } \\
\text { Wise pattern } \\
\text { Batwing }\end{array}$ & $\begin{array}{l}0(0 \%) \\
1(5 \%)\end{array}$ & $\begin{array}{l}1(5 \%) \\
4(20 \%)\end{array}$ & $\begin{array}{l}6(30 \%) \\
7(35 \%)\end{array}$ & $\begin{array}{l}9(45 \%) \\
7(35 \%)\end{array}$ & $\begin{array}{l}4(40 \%) \\
15(5 \%)\end{array}$ & $\begin{array}{l}23.75 \\
17.25\end{array}$ & 135 & 0.065 \\
\hline
\end{tabular}


This table shows: According to physician questionnaire, there were significant differences in assessment of scar visibility and breast projection between the two operations in the favor of wise pattern.

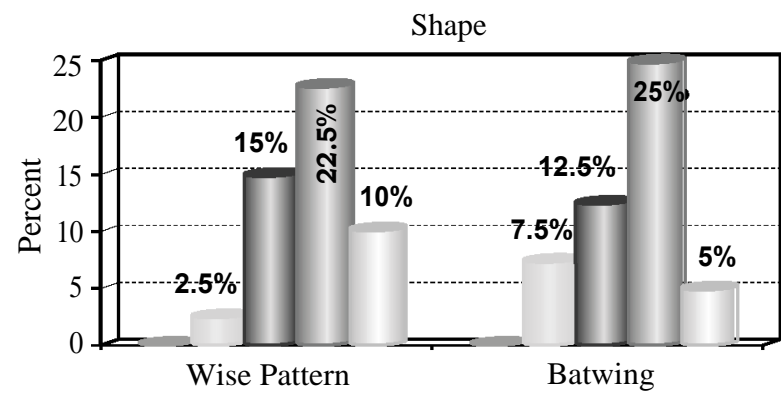

Mamoplastic Surgery

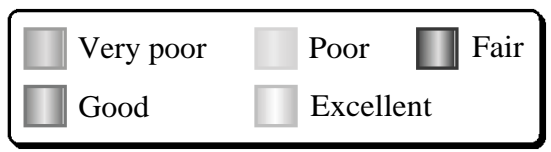

Fig. (19): This figure shows the post-operative assessment of the shape of the breast for both operations.

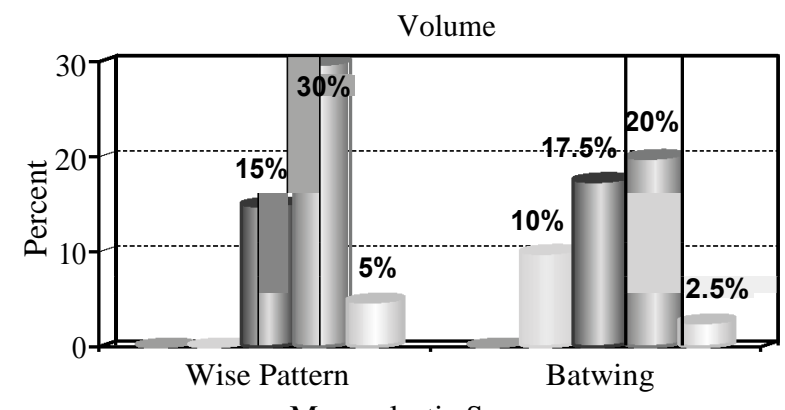

Mamoplastic Surgery

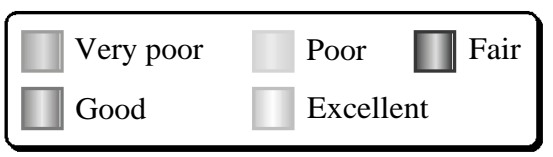

Fig. (20): This figure shows the post-operative assessment of the volume of the breast for both operation.
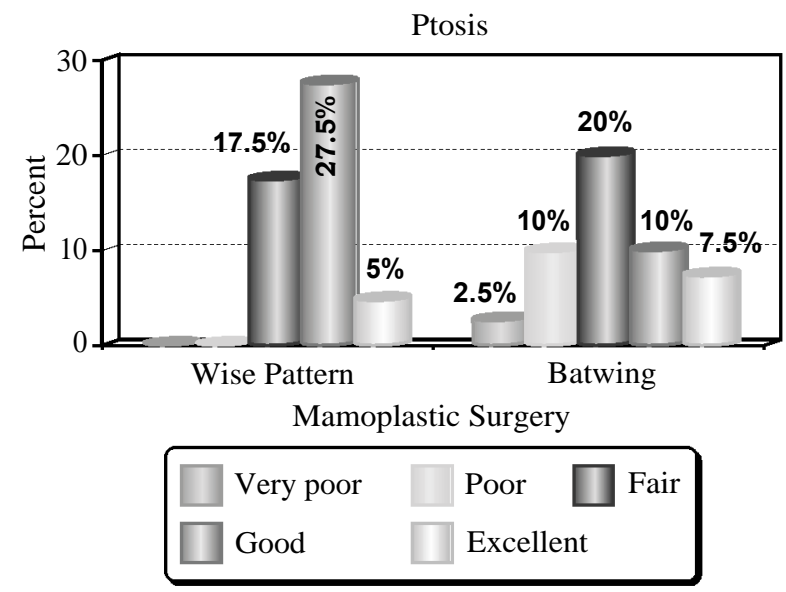

Fig. (21): This figure shows the post-operative assessment of ptosis of the breast for both operations.

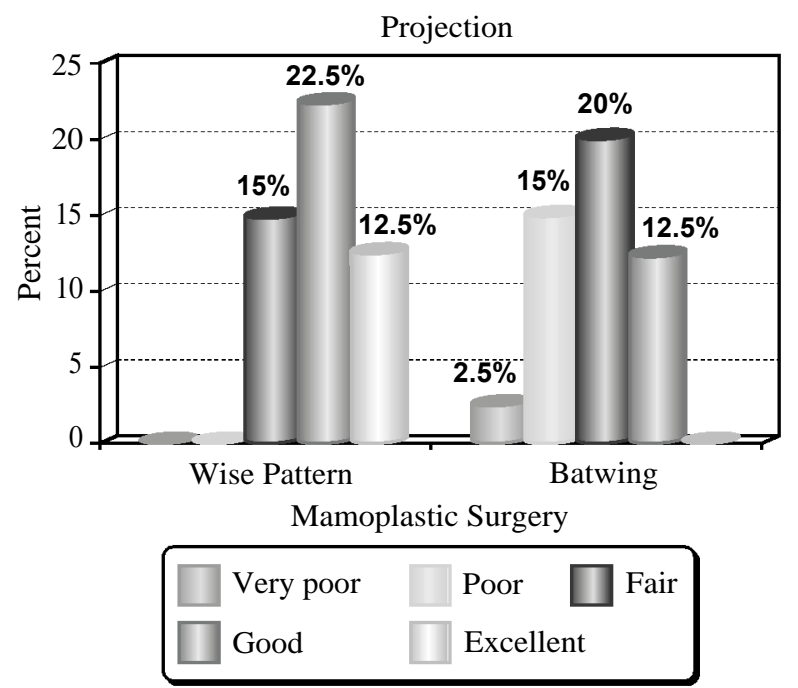

Fig. (22): This figure shows the post-operative assessment of breast projection for both operations.

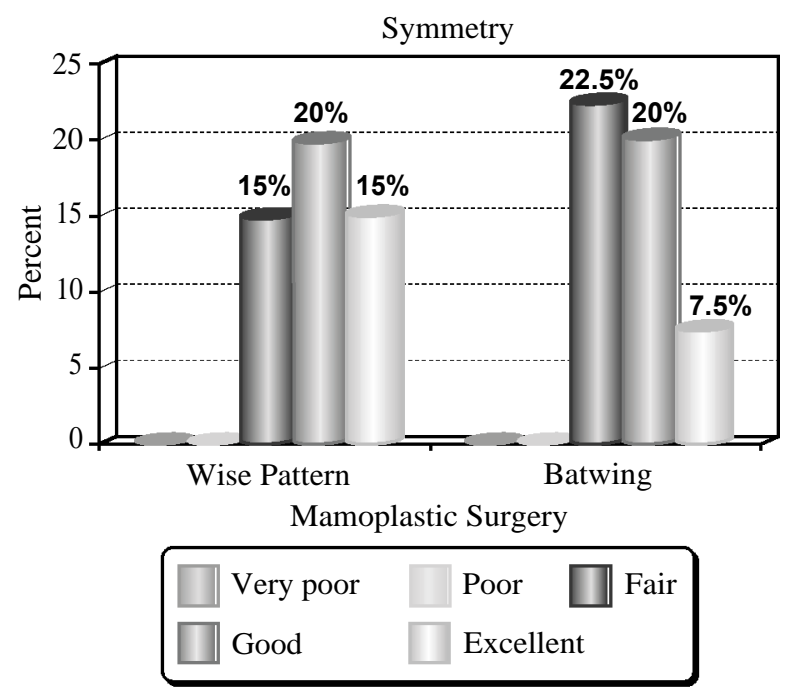

Fig. (23): This figure shows the post-operative assessment of breast symmetry for both operations.

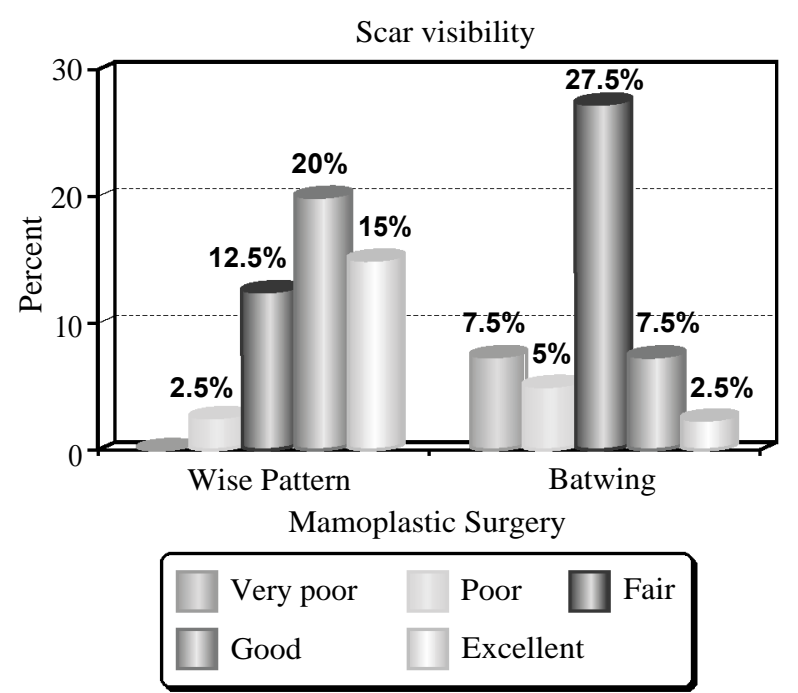

Fig. (24): This figure shows the post-operative assessment of scar visibility for both operations. 


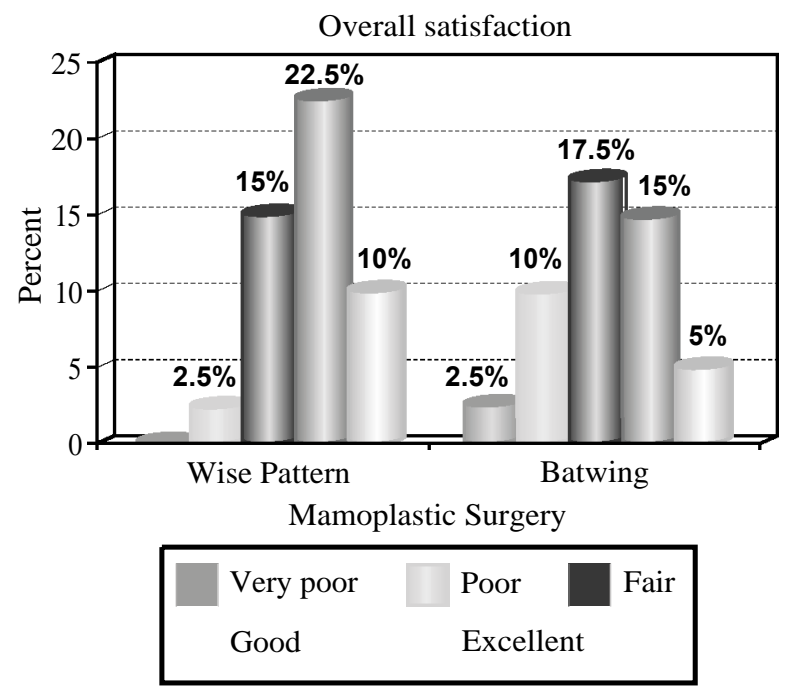

Fig. (25): This figure shows the post-operative assessment of overall satisfaction of the patient for both operations.

\section{Discussion}

Breast cancer is the most common cancer diagnosed and the second most common cause of cancer-related mortality among women. Currently, the lifetime risk of breast cancer among women is 1 in 8 or $12 \%$ compared to 1 in 11 for women in the 1970s [10]

The majority of breast malignancies present in the central and upper quadrants of the breast. Surgeons involved in the management of breast cancer should have a range of surgical options for resecting upper pole lesions that preserve or enhance the aesthetic or minimize defects following breast cancer surgery [11].

Upper pole tumours of the breast form a challenge to some extent to the oncoplastic surgeon, especially when considering the aesthetics of the breast. Scars in this area are very unsightly. Although batwing and wise pattern mammoplasty are described for the same tumour location, many differences exist between the two approaches [12]

In addition to physical preservation, women who undergo breast conservation have a better view of their body image, are more comfortable with their shape, and might have less adverse physical sequelae from asymmetry, chest wall adhesions, and numbness associated with mastectomy [13].

A successful oncoplastic procedure begins with selecting the appropriate operation for a given patient, which takes into account a patient's unique breast anatomy (e.g., breast shape and degree of ptosis). An understanding of tumor location and extent, as well as appreciation of the patient's goals [14].

The aim of our study is to compare between batwing mammoplasty and inferiorly based wise pattern therapeutic mammoplasty in management of upper pole breast tumors regarding cosmetic results, oncological outcomes, rate of complications and degree of satisfaction of the patients.

As regard to demographic data of the current study, 40 patients were included in this study. The mean age of our wise pattern participants was (45.7) and the mean age of our batwing participants was (44.6) years this is in line with Shin et al., [15] who found that the mean age of breast cancer participants was (45.7) years. But lower than Malik et al., [16] who found the mean age of breast cancer patients was $(53.1 \pm 11.5)$ years.

Breast cancer incidence increases with age, as does the incidence of many other chronic diseases, such as diabetes, hypertension, and Cardiovascular Disease (CVD); hence, many breast cancer patients have one or more comorbid medical conditions at diagnosis. Comorbidities have been shown to influence cancer treatment decisions and short-and long-term survival [17].

In our study we had (5\%) of patients suffered from hypertension, $(5 \%)$ of patients suffered from diabetes and (5\%) of patients had asthma this is in according with Nechuta et al., [18] who found that the prevalence of diabetes mellitus was $(6.2 \%)$, chronic bronchitis/asthma (5.8\%) and hypertension $(5.0 \%)$.

The ptotic breast is characterized by the inferolateral descent of both the glandular breast and the nipple-areolar complex. In the early stages of ptosis, the nipple-areolar complex and gland descend at the same rate. More advanced stages of breast ptosis are marked by a nipple-areolar complex descent out of proportion to the glandular descent [19]

In our study all the participants had ptosis. In wise pattern operation participants (10\%) had grade 1 ptosis, (70\%) had grade 2 ptosis, $(20 \%)$ had grade 3 ptosis while in batwing participants (5\%) had grade 1 ptosis, (75\%) had grade 2 ptosis, (20\%) had grade 3 ptosis. This is in line with Foustanos et al., [20] who stated that $(8.5 \%)$ were grade 1 , (76.1\%) of participants were grade 2 and $(19.4 \%)$ were grade 3 .

Invasive Ductal Carcinoma (IDC) is the most common type of breast cancer. About $80 \%$ of all 
breast cancers are invasive ductal carcinomas. Invasive lobular breast cancer is the second most common type of breast cancer. Over $10 \%$ of invasive breast cancers are invasive lobular carcinomas [21].

In this study, (90\%) in wise pattern group and (95\%) of batwing group had IDC while (10\%) in wise pattern group and $(5 \%)$ of batwing group had ILC. This in line with Giuliano et al., [22] who evaluated invasive lobular and ductal carcinoma incidence rates and stated that the incidence of IDC ranged from (85\%-97\%) and ILC incidence ranged from $(7.6 \%-14.9 \%)$. But our results were higher than Tonellotto et al., [23] who found that the incidence of IDC was (87.4\%) and ILC incidence was $(5.7 \%)$.

TNM staging system, provides a flexible platform for prognostic classification based on traditional anatomic factors, which can be modified and enhanced using patient biomarkers and multifactorial prognostic panel data [24].

In this study there were (2) patients in T1N0 stage, (2) patient in T1N1 stage, (5) patients in T2N0 stage and (11) patients in T2N1 stage in wise pattern group while in batwing group there were (1) patient in T $1 \mathrm{~N} 0$ stage, (3) patient in T $1 \mathrm{~N} 1$ stage, (3) patients in T2N0 stage and (13) patients in $\mathrm{T} 2 \mathrm{~N} 1$ stage.

There was significant difference in the time of operation between wise pattern and batwing groups where the mean operation time in batwing was $(104 \pm 18.25 \mathrm{~min})$ this is in according with Hashem and Farahat [12] who found that the mean operative time in batwing was $103 \mathrm{~min}$. Also, in line with Grubnik et al., [25] who found that the mean operative time in batwing was $102 \mathrm{~min}$.

As regard to wise pattern group the mean operation time was $(192.3 \pm 14.72 \mathrm{~min})$ which is higher than Hashem and Farahat [12] who stated that the mean operative time is $220 \mathrm{~min}$ in wise pattern group.

As regard to hospital stay after operation the mean hospital stay in batwing $(2.4 \pm 0.82)$ days and the mean hospital stay in wise pattern was $(2.2 \pm$ 0.52 ) days this is in line with Mohsen and Marzouk [26] who found that the mean hospital stay after mastopexy was $(3.5 \pm 1.06)$ days.

Speaking about the weight range of breast tissue excised was (573-859) grams in batwing and (504791) grams in wise pattern this is in line with (Grubnik et al., [25]) who found that the range of breast tissue excised from the affected breast was $(17-1,316 \mathrm{~g})$.

Surgical wound complications remain a major cause of morbidity, leading to higher costs and reduced quality of life [27].

In the present study (5\%) patient from each group presented with gapped wound this is in according with DellaCroce et al., [28] who stated that $(6.4 \%)$ suffered from partial incisional dehiscence. There was $(5 \%)$ of the patients in wise pattern group had an infected wound this is in line with Cho et al., [29] who reported that infection rates of only $3-7 \%$.

In his study (10\%) of the patients in each group suffered from seroma after operation this is in line with Gonzalez et al., [30] who found that incidence of seroma is (10\%) in patients with $1 \mathrm{~cm}$ tumor.

Partial or total nipple necrosis after breast reduction surgery can be a devastating complication for the patient and the surgeon. Frequent monitoring of the Nipple-Areola Complex (NAC) and early identification of vascular compromise followed by appropriate action may prevent total NAC loss [31].

NAC necrosis has been reported in $2 \%$ of breast reduction cases and in $1 \%$ of mastopexy cases; epidermolysis with blister like formation owing to intradermal or subdermal edema may result in 5\% to $11 \%$ of cases [32]

In our study there was no significant difference between wise pattern group and batwing group regarding nipple and areola vasculature and sensation. (10\%) of patients in wise pattern group reported vasculature affection of nipple and areola complex while no cases were reported in batwing group. $(10 \%)$ of patients in wise pattern group reported loss of sensation of nipple and areola complex while no cases in batwing group this is in according with Spear et al., [33] who found that nipple and areola complication after reduction mammoplasty were $(9.5 \%)$.

Breast Cancer (BC) treatment leads to mutilation and destruction of breast shape with negative effects on body image and self-esteem. One of the main goals of reconstructive and oncoplastic breast surgery is to satisfy patients and improve their quality of life [34].

Therefore, it is important to assess the patient experience post-surgery by means of PatientReported Outcome Measures (PROMs) that focus on the patient's perception of the surgery and 
surgical care, as well as psychosocial well-being and physical functioning [34]

In this study we compared the results of batwing mammoplasty and wise pattern mammoplasty in the surgical management of upper pole breast tumors.

Both surgeries were assessed by the general surgeon and the patient. The patients were rated for breast symmetry, shape, volume, ptosis, projection, symmetry, scar visibility, overall satisfaction. We used a scale where in the overall results were rated from 5 to 1 ( $5=$ excellent, $4=$ good, 3 = fair, 2 = poor, $1=$ very poor $)$.

As regard to the patient assessment of cosmetic output there were significant differences between the two operations in scar visibility, breast projection and overall satisfaction in favor of wise pattern operation this is in line with Grubnik et al., [25] who used nonmedical personnel to assess the photos of the patient and there wassignificant differences between the two operations in scar visibility, breast projection and overall satisfaction in favor of wise pattern operation.

According to physician questionnaire, there were significant differences in assessment of scar visibility and breast projection between the two operations in the favor of wise pattern this is in line with Grubnik et al., [25] who used a general surgeon and a plastic surgeon to assess the photos of the patient and there were significant differences in assessment of scar visibility and breast projection between the two operations in the favor of wise pattern.

In this study according to the patients the overall satisfaction in wise pattern operation was $(0 \%)$ very poor, (15\%) poor, (40\%) fair, (35\%) good and $(10 \%)$ excellent while in batwing operation $(0 \%)$ very poor, $(5 \%)$ poor, $(15 \%)$ fair, $(55 \%)$ good and (25\%) excellent. Also, according to physician the overall satisfaction in wise pattern operation was $(0 \%)$ very poor, $(5 \%)$ poor, $(30 \%)$ fair, $(45 \%)$ good and $(20 \%)$ excellent while in batwing operation $(5 \%)$ very poor, $(20 \%)$ poor, $(35 \%)$ fair, $(35 \%)$ good and (5\%) excellent.

In this study the overall satisfaction was in favor of wise pattern operation this is in line with Hashem and Farahat [12] who found that the overall cosmetic results between batwing and wise pattern was in favor of wise pattern operation.

In light of the experience displayed in this study, the batwing mammoplasty technique has proven to be a simple and quick procedure for upper pole breast tumors. It will result in an acceptable cosmetic result with a relatively low risk of post-operative complications when compared to wise pattern therapeutic mammoplasty.

On the other hand, wise pattern therapeutic mammoplasty remains a more cosmetically appealing techniquethat achieves superior aesthetic outcome. It is associated, however, with more complications and a risk of some degree of sensory loss over nipple and areola. So it would be more suitable for younger patients with no significant medical co-morbidities and patients who put significant value on their cosmetic result.

\section{Conclusion:}

In this study, we concluded that: Both techniques batwing mammoplasty and wise pattern therapeutic mammoplasty are valid options for upper pole breast tumors. Wise pattern therapeutic mammoplasty remains aesthetically superior; however, batwing mammoplasty is an easy, simple technique with acceptable results to patients.

\section{Recommendations:}

From this study, we could recommend:

1- The decision of which surgical approach to be used for the oncoplastic procedure is heavily based on patient and tumor characteristics.

2- The pre-operative evaluation should include examination for degree of ptosis, overall skin quality, evidence of prior radiation, and overall breast size.

3- Successful oncoplastic procedure begins with selecting the appropriate operation for a given patient, which takes into account a patient's unique breast anatomy (e.g., breast shape and degree of ptosis) and good understanding of tumor location and extent, as well as appreciation of the patient's goals.

4- Mastectomy with reconstruction may provide a more aesthetically pleasing result than breast conservation surgery in the small to moderatebreasted woman.

5- Larger breasted women have more options available for reconstruction, whether it is local tissue rearrangement, local or regional flaps, or reduction mammoplasty/mastopexy.

6- Batwing mammoplasty procedure achieve optimal results (i.e., breast contour and nipple projection) in patients with larger breast volume and a mild to moderate degree of breast ptosis. 
7- More studies on a large scale should be performed to assess the results of batwing mammoplasty and wise pattern therapeutic mammoplasty regarding complication and cosmetic outcome for management of upper pole breast tumours.

\section{References}

1- KOÇAN S. and GÜRSOY A.: Body image of women with breast cancer after mastectomy: A qualitative research. The Journal of Breast Health, 12 (4): 145, 2016.

2- KARAMANOU M., A. DIAMADIS and G. ANDROUTSOS: "The first cases of cancer in antiquity." Journal of BU ON.: Official Journal of the Balkan Union of Oncology, 13 (4): 601-8, 2008.

3- SHARMA G.N., R. DAVE, J. SANADYA, P. SHARMA and K. SHARMA: "Various types and management of breast cancer: An overview." Journal of Advanced Pharmaceutical Technology \& Research, 1 (2): 109-20, 2010.

4- AL SALEH S., F. AL MULLA and Y.A. LUQMANI: "Estrogen receptor silencing induces epithelial to mesenchymal transition in human breast cancer cells." PloS one, 6 (6): 83-95, 2011.

5- SCAGLIONE S.: "Early Detection and Prevention of Breast Cancer in Women." J. Womens Health Care Manage, 1: 1-20, 2020.

6- SHAH R., K. ROSSO and S.D. NATHANSON: "Pathogenesis, prevention, diagnosis and treatment of breast cancer." World Journal of Clinical Oncology, 5 (3): 283, 2014.

7- CLOUGH K.B., G.J. KAUFMAN, C. NOS, I. BUCCIMAZZA and I.M. SARFATI: "Improving breast cancer surgery: A classification and quadrant per quadrant atlas for oncoplastic surgery." Annals of Surgical Oncology, 17 (5): 1375-91, 2010.

8- HERSHMAN D.L., D. BUONO, J.S. JACOBSON, R.B McBRIDE, W.Y. TSAI, K.A. JOSEPH, et al.: "Surgeon characteristics and use of breast conservation surgery in women with early stage breast cancer." Annals of surgery, 249 (5), 2009.

9- ELKHATEB M.A., A.A. SHMILLA, S.A. EL-SHAKHS, A.M. AL-BARAH, A.F. MOHAMED and M.A. MOHAMMED: "Oncoplastic breast-conserving surgery." Menoufia Medical Journal, 28 (1): 49-65, 2015.

10- YOULDEN D.R., S.M. CRAMB, C.H. YIP and P.D. BAADE: "Incidence and mortality of female breast cancer in the Asia-Pacific region." Cancer biology \& medicine, 11 (2): 101-20, 2014

11-HOLMES D.R.: Batwing and Hemibatwing Mammoplasty. Oncoplastic Breast Surgery Techniques for the General Surgeon, Springer: 175-86, 2020.

12- HASHEM T. and A. FARAHAT: "Batwing versus Wise pattern mammoplasty for upper pole breast tumours: A detailed comparison of cosmetic outcome." World Journal of Surgical Oncology, 15 (1): 60, 2017.

13-ANDERSON B.O., R. MASETTI and M.J. SILVERSTEIN: "Oncoplastic approaches to partial mastectomy: An overview of volume-displacement techniques." The lancet oncology, 6 (3): 145-57, 2005.
14- MAXWELL V.K., MALIK A., P.A. JEYARAJ, A. SHANKAR, G.K. RATH, S. MUKHOPADHYAY, VG. P., T. STORM-DICKERSON, P. WHITWORTH, C. RUBANO and A. GABRIEL: "Advances in nipple-sparing mastectomy: Oncological safety and incision selection." Aesthetic Surgery Journal, 31 (3): 310-9, 2011.

15- SHIN S.U., N. CHO, H.B. LEE, S.Y. KIM, A. YI, S.Y. KIM, et al.: "Neoadjuvant chemotherapy and surgery for breast cancer: Pre-operative MRI features associated with local recurrence." Radiology, 289 (1): 30-8, 2018.

16- MALIK A., P.A. JEYARAJ, A. SHANKAR, G.K. RATH, S. MUKHOPADHYAY, V.K. MAXWELL, G.P., T. STORM-DICKERSON, P. WHITWORTH, C. RUBANO and A. GABRIEL: "Advances in nipple-sparing mastectomy: Oncological safety and incision selection." Aesthetic Surgery Journal, 31 (3): 310-9, 2011.

17- LAND L.H., S.O. DALTON, T.L. JØRGENSEN and M. EWERTZ: "Comorbidity and survival after early breast cancer. A review." Critical reviews in oncology/ hematology, 81 (2): 196-205, 2012.

18- NECHUTA S., W. LU, Y. ZHENG, H. CAI, P.P. BAO, K. GU, et al.: "Comorbidities and breast cancer survival: A report from the Shanghai Breast Cancer Survival Study." Breast cancer research and treatment, 139 (1): 227-35, 2013.

19- WHITE D.J. and G.P. MAXWELL: Reduction mammaplasty. Plastic Surgery Secrets Plus, Mosby: 446-52, 2010.

20- FOUSTANOS A., K. PANAGIOTOPOULOS and G. SKOURAS: "Intraoperative modification of Pitanguy technique of reduction mammaplasty for elevation of the nipple-areola complex in case of severe breast ptosis." Aesthetic Plastic Surgery, 35 (1): 55-60, 2011.

21- ARPS D.P., P. HEALY, L. ZHAO, C.G. KLEER and J.C. PANG: "Invasive ductal carcinoma with lobular features: A comparison study to invasive ductal and invasive lobular carcinomas of the breast." Breast cancer research and treatment, 138 (3): 719-26, 2013.

22- GIULIANO A.E., K.K. HUNT, K.V. BALLMAN, P.D. BEITSCH, P.W. WHITWORTH, P.W. BLUMENCRANZ, et al.: "Axillary dissection vs no axillary dissection in women with invasive breast cancer and sentinel node metastasis: A randomized clinical trial." Jama, 305 (6): 569-75, 2011

23- TONELLOTTO F., A. BERGMANN K. De SOUZA ABRAHÃO, S.S. De AGUIAR, M.A. BELLO and L.C.S. THULER: "Impact of number of positive lymph nodes and lymph node ratio on survival of women with nodepositive breast cancer." European Journal of Breast Health, 15 (2): 76, 2019.

24- HORTOBAGYI G.N., S.B. EDGE and A. GIULIANO: "New and important changes in the TNM staging system for breast cancer." American Society of Clinical Oncology Educational Book, 38: 457-67, 2018.

25- GRUBNIK A., C. BENN and G. EDWARDS: "Therapeutic mammaplasty for breast cancer: Oncological and aesthetic outcomes." World Journal of Surgery, 37 (1): 72-83, 2013.

26- MOHSEN S.M. and M. MARZOUK: "Round block technique in the management of early stages of breast cancer: An assessment of the technique, oncological safety, and cosmetic outcomes." The Egyptian Journal of Surgery, 37 (3): 271, 2018. 
27- BRITTEON P., N. CULLUM and M. SUTTON: "Association between psychological health and wound complications after surgery." British Journal of Surgery, 104 (6): 769-76, 2017.

28- DELLACROCE F.J., C.A. BLUM, S.K. SULLIVAN, A. STOLIER, C. TRAHAN, M.W. WISE, et al.: "Nipplesparing mastectomy and ptosis: Perforator flap breast reconstruction allows full secondary mastopexy with complete nipple areolar repositioning." Plastic and Reconstructive Surgery, 136 (1): 1, 2015.

29- CHO J., S. SHIN, J. PARK, Y. SONG, J. YI, M. PARK, et al.: "Analysis of infections occurring in breast cancer patients after breast conserving surgery using mesh." Journal of Breast Cancer, 14 (4): 328-32, 2011.

30- GONZALEZ E.A., E.C. SALTZSTEIN, C.S. RIEDNER and B.K. NELSON: "Seroma formation following breast cancer surgery." The Breast Journal, 9 (5): 385-8, 2003.

31- WONG C., M. VUCOVICH and R. ROHRICH: "Mast- opexy and reduction mammoplasty pedicles and skin resection patterns." Plastic and Reconstructive Surgery Global Open, 2 (8): 5-19, 2014.

32- RANCATI A., M. IRIGO and C. ANGRIGIANI: "Management of the Ischemic Nipple-Areola Complex After Breast Reduction." Clinics in Plastic Surgery, 43 (2): 403 14, 2016.

33- SPEAR S.L., S.C. WILLEY, E.D. FELDMAN, C. COCILOVO, M. SIDAWY, A. AL-ATTAR, et al.: "Nipplesparing mastectomy for prophylactic and therapeutic indications." Plastic and Reconstructive Surgery, 128 (5): 1005-14, 2011.

34- SHEKHAWAT L., L. BUSHERI S. DIXIT C. PATEL U. DHAR and C. KOPPIKER: "Patient-reported outcomes following breast reconstruction surgery and therapeutic mammoplasty: Prospective evaluation 1 year post-surgery with BREAST-Q questionnaire." Indian Journal of Surgical Oncology, 6 (4): 356-62, 2015.

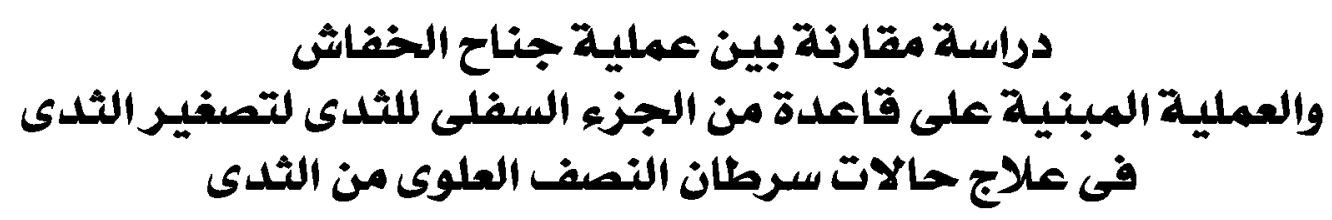

يعد سرطان الثدى آكثر آنواع السرطان شيوعاً بين النساء وثانى آكثر آسباب الوفيات المرتبطة بالسرطان. ويمكن آن يصيب سرطان

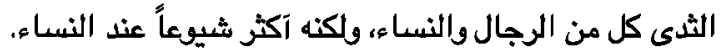

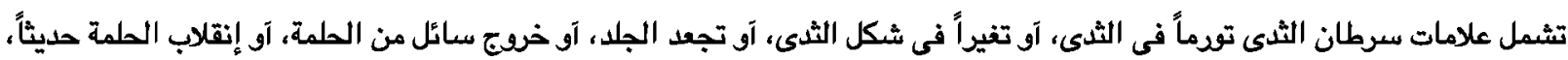

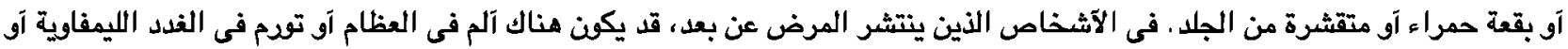
ضيق فى التنفس.

تُعرف السرطانات التى تتطود من القنوات بإسم سرطان الآتنية، بينما تُعرف السرطانات التى تنشاً من الفصيصات بالسرطان الفصيصى. الهدف من دراستنا هو إجراء مقارنة بين عملية جناح الخفاش والعملية المبنية على قاعدة من الجزء السفلى اللثى لتصفير الثدى فى علاج

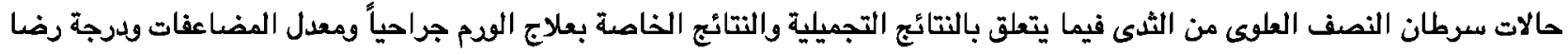
المرضى' • لقد آثبتت تقنية تجميل الثدى بجناح الخفاش آنها إجراء بسيط وسريع لآورام الثدى فى العمو العلوى.

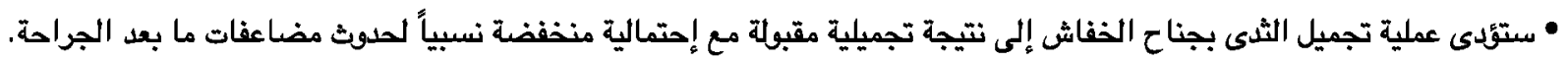
• إجراء الخفاش ليس مناسباً فقط للمرضى والكنه مناسب جداً للجراحين فى بداية حياتهم المهنية فى جراحة الآهدام إنها طريقة جراحية سهلة إلى حد ما مع نتائج مرضية اللغاية.

• يمكن دمج خط خياطة جراحة تجميل الثدى بجناح الخفاش بسهولة فى خط شق إستئصال الثدى فى المستقبل فى حالات إرتجاع الودم. • تظل العملية المبنية على قاعدة من الجزء السفلى الثدى لتصغير الثدى آسلوباً آكثر جاذبية من الناحية التجميلية يحقق نتائج جمالية فائقة.

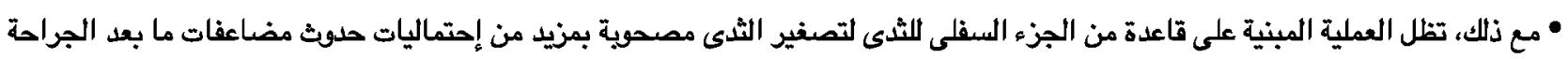

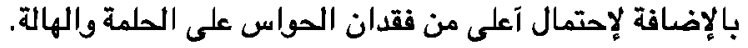

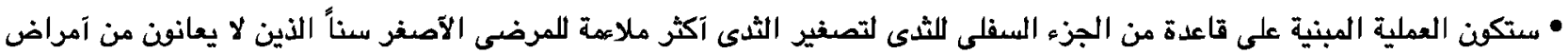

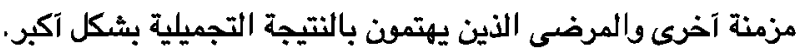

